\title{
A posteriori error estimates for finite volume approximations of Elliptic Equations on General Surfaces
}

\author{
Lili $\mathrm{Ju}^{1, *}$ \\ Department of Mathematics, University of South Carolina, Columbia, SC 29208, \\ $U S A$. \\ Li Tian \\ Department of Mathematics, University of South Carolina, Columbia, SC 29208, \\ $U S A$. \\ Desheng Wang ${ }^{2}$ \\ Division of Mathematical Sciences, School of Physical 86 Mathematical Sciences, \\ Nanyang Technological University, 637616, Singapore
}

\begin{abstract}
In this paper, we present a residual-based a posteriori error estimate for the finite volume discretization of steady convection-diffusion-reaction equations defined on surfaces in $\mathbb{R}^{3}$, which are often implicitly represented as level sets of smooth functions. Reliability and efficiency of the proposed a posteriori error estimator are rigorously proved. Numerical experiments are also conducted to verify the theoretical results and demonstrate the robustness of the error estimator.
\end{abstract}

Key words: A posteriori error estimates, finite volume approximation, PDEs on surfaces

\footnotetext{
* Corresponding author.

Email addresses: ju@math.sc.edu (Lili Ju), tianl@mailbox.sc.edu (Li Tian), desheng@ntu.edu.sg (Desheng Wang).

1 This author's research is supported in part by the National Science Foundation under grant number DMS-0609575 and the Department of Energy under grant number DE-FG02-07ER64431.

2 The author's research is partially supported by the National Research Foundation of Singapore under the grant number NRF2007IDM-IDM002-010 and the Ministry of Education under the grant number M58110011.
} 


\section{Introduction}

In the area of numerical solution of partial differential equations (PDEs), $a$ posteriori error estimates are computable quantities in terms of the approximate solutions, and provide a reliable and efficient measurement for the errors of the discrete solution without knowing the exact solution. Reliability is often referred to as the property that the true error can be bounded from above by the error estimator (up to some constant) and efficiency implies that the true error is also locally bounded from below by the error estimator. A posteriori error estimates have played a very important role in adaptive mesh generation and algorithm design for numerical PDEs. Theoretical and systematical study of a posteriori error estimators for finite element approximation began in the late 1970's [5], and since then a lot of literature has gradually appeared, see $[2,4,6,7,9,11,14,29,40]$ and the references cited therein. We would like to point out that elegant analysis on residual-based a posteriori estimates of finite volume approximations for elliptic equations can be found in $[1,12]$.

Numerical solution of PDEs defined on smooth surfaces (or manifolds) in $\mathbb{R}^{3}$ recently attracted a lot of attention due to its applications in various areas, such as surface diffusion, global and local geophysical flows, ice formation, brain warping and so on $[27,33,34,35]$. Thus it is of interest to investigate useful a posteriori error estimates for these types of problems. A lot of a priori error analysis for second-order and fourth-order problems have been done for finite element methods [22, 23, 24] and finite volume methods [8, 20, 19]. Recently, a posteriori error estimates of finite element methods for discretizing the Laplace-Beltrami operator on surfaces were rigorously analyzed in $[3,15$, 28] while similar studies for finite volume methods are currently lacking as far as we know. In this paper, we will rigorously derive a residual-based explicit a posteriori error estimator (in the sense of energy norm) for the finite volume discretization of the elliptic equations defined on a smooth surface which is represented as the zero level set of a signed distance function $d$ to the surface.

The paper is organized as follows: in Section 1.1, we will briefly review the model problem defined on surfaces and define some notations to facilitate our analysis. Then a finite volume discretization (generalized central scheme) of the problem is given in Section 2. In Section 3, we will derive a residualbased a posteriori estimate for the discretization in terms of the approximate solution, and prove its reliability and efficiency. Some numerical experiments are included in Section 4, to verify the theoretical results. In addition, we also numerically demonstrate that the derived a posteriori error estimator is quite robust, i.e., the constants in the a posteriori estimates are almost uniform across all test problems. Finally, concluding remarks are given in Section 5. 


\subsection{Model problem}

Let $\mathbf{S}$ in $\mathbb{R}^{3}$ be an open bounded $C^{k, \alpha}$-hypersurface $[22,26]$ with $k \in \mathbb{N} \cup\{0\}$ and $0 \leq \alpha<1$, and we assume that $\mathbf{S}$ is represented globally by some oriented distance function (or say level set function) $d=d(\mathbf{x})$ defined in some open subset $\mathbf{U}$ of $\mathbb{R}^{3}$ such that $\mathbf{S}=\{\mathbf{x} \in \mathbf{U} \mid d(\mathbf{x})=0\}$ with $d \in C^{k, \alpha}$ and $\nabla d \neq 0$. The unit outward normal to $\mathbf{S}$ (with increasing $d$ ) at $\mathbf{x}$ is given by

$$
\overrightarrow{\mathbf{n}}(\mathbf{x})=\left(n_{1}(\mathbf{x}), n_{2}(\mathbf{x}), n_{3}(\mathbf{x})\right)=\frac{\nabla d(\mathbf{x})}{|\nabla d(\mathbf{x})|}
$$

where $|\cdot|$ denotes the Euclidean norm and $\nabla$ denotes the standard gradient operator in $\mathbb{R}^{3}$. Without loss of generality, we assume that $|\nabla d| \equiv 1$.

Let $\nabla_{s}=\left(\nabla_{s, 1}, \nabla_{s, 2}, \nabla_{s, 3}\right)=\nabla-\overrightarrow{\mathbf{n}}(\overrightarrow{\mathbf{n}} \cdot \nabla)$ denote the tangential (surface) gradient operator, $\Delta_{s}=\nabla_{s} \cdot \nabla_{s}$ is then called the Laplace-Beltrami operator associated with $\mathbf{S}[26]$. We use the standard notation for Sobolev spaces $L^{p}(\mathbf{S})$, $W^{m, p}(\mathbf{S})$, and $H^{m}(\mathbf{S})=W^{m, 2}(\mathbf{S})$ on $\mathbf{S}$. To make space $H^{m}(\mathbf{S})$ well defined, it is customary to assume $k+\alpha \geq \max \{1, m\}$, see [32]. To avoid technical complexities, we further assume that $\mathbf{S}$ and $\partial \mathbf{S}$ are sufficiently smooth (say, of class $C^{3}$ ) and $\partial \mathbf{S} \neq \varnothing$ for the rest of the paper unless stated otherwise.

We consider the following steady diffusion-convection-reaction equation imposed on $\mathbf{S}$,

$$
-\nabla_{s} \cdot\left(a(\mathbf{x}) \nabla_{s} u(\mathbf{x})\right)+\nabla_{s} \cdot(\overrightarrow{\mathbf{v}}(\mathbf{x}) u(\mathbf{x}))+b(\mathbf{x}) u(\mathbf{x})=f(\mathbf{x}) \quad \forall \mathbf{x} \in \mathbf{S},
$$

where the data in (1) is assumed to satisfy:

Assumption $1 f \in L^{2}(\mathbf{S})$, a $(\mathbf{x})$ is uniformly continuous on $\mathbf{S}, \overrightarrow{\mathbf{v}} \in\left(W^{1, \infty}(\mathbf{S})\right)^{3}$, and $b \in L^{\infty}(\mathbf{S})$. Additionally, $a(\mathbf{x}) \geq \alpha_{1}>0, b(\mathbf{x}) \geq 0$ and $\nabla_{s} \cdot \overrightarrow{\mathbf{v}}(\mathbf{x}) / 2+b(\mathbf{x}) \geq$ $\alpha_{2} \geq 0$ for any $\mathbf{x} \in \mathbf{S}$.

For simplicity, we take the homogeneous Dirichlet boundary condition:

$$
u(\mathbf{x})=0, \quad \forall \mathbf{x} \in \partial \mathbf{S}
$$

Note that our discussion here can be extended to more general cases such as $a=a(\mathbf{x})$ being a symmetric positive-definite tensor.

For any $u, \phi \in H_{0}^{1}(\mathbf{S})$, define the bilinear functional $\mathcal{A}$ to be

$$
\mathcal{A}(u, \phi)=\int_{\mathbf{S}} a \nabla_{s} u \cdot \nabla_{s} \phi d s-\int_{\mathbf{S}} u \overrightarrow{\mathbf{v}} \cdot \nabla_{s} \phi d s+\int_{\mathbf{S}} b u \phi d s .
$$

Based on Assumption 1, we have (for some generic constants $c_{1}>0$ and 
$\left.c_{2}>0\right)$

$$
\begin{aligned}
& \mathcal{A}(u, \phi) \leq c_{1}\|u\|_{H^{1}(\mathbf{S})}\|\phi\|_{H^{1}(\mathbf{S})}, \\
& \mathcal{A}(u, u) \geq c_{2}\|u\|_{H^{1}(\mathbf{S})}^{2} .
\end{aligned}
$$

The above two conditions guarantee that the expression $\mathcal{A}(u, u)$ is equivalent to the norm in $H_{0}^{1}(\mathbf{S})$ under given assumptions. We then define the so-called "energy" norm as $\|u\|_{E}=\sqrt{\mathcal{A}(u, u)}[12,30]$.

We say that $u \in H_{0}^{1}(\mathbf{S})$ is a weak solution of the equation (1) if and only if

$$
\mathcal{A}(u, \phi)=(f, \phi)_{s} \quad \forall \phi \in H_{0}^{1}(\mathbf{S}),
$$

where

$$
(f, \phi)_{s}=\int_{\mathbf{S}} f(\mathbf{x}) \phi(\mathbf{x}) d s .
$$

The existence of the weak solution of equation (1) under Assumption 1 follows from the standard elliptic equation theory [22, 26].

Theorem 2 Under Assumption 1, there exists a unique weak solution $u \in$ $H_{0}^{1}(\mathbf{S})$ of (1). Moreover, $u \in H^{2}(\mathbf{S})$ and satisfies that

$$
\|u\|_{H^{2}(\mathbf{S})} \leq c\|f\|_{L^{2}(\mathbf{S})} .
$$

for some generic constant $c>0$.

We note that in the case of $\partial \mathbf{S}=\varnothing$, one can also show that, if $\alpha_{2}>0$ in Assumption 1, then there exists a unique weak solution $u \in H^{1}(\mathbf{S})$ of (1).

\section{Finite volume discretization}

\subsection{Piecewise linear approximation of the surface}

We assume that $\mathbf{S}$ is a connected compact smooth hypersurface which is the zero level set of a signed distance function $|d(\mathbf{x})|=\operatorname{dist}(\mathbf{x}, \mathbf{S})$ defined on a strip (band)

$$
\mathbf{U}=\left\{\mathbf{x} \in \mathbb{R}^{3} \quad \mid \quad \operatorname{dist}(\mathbf{x}, \mathbf{S})<\delta\right\}, \quad \text { for some } \delta>0,
$$

around $\mathbf{S}$ such that there is a unique decomposition for any $\mathbf{x} \in \mathbf{U}$,

$$
\mathbf{x}=\mathbf{p}(\mathbf{x})+d(\mathbf{x}) \overrightarrow{\mathbf{n}}(\mathbf{x})
$$



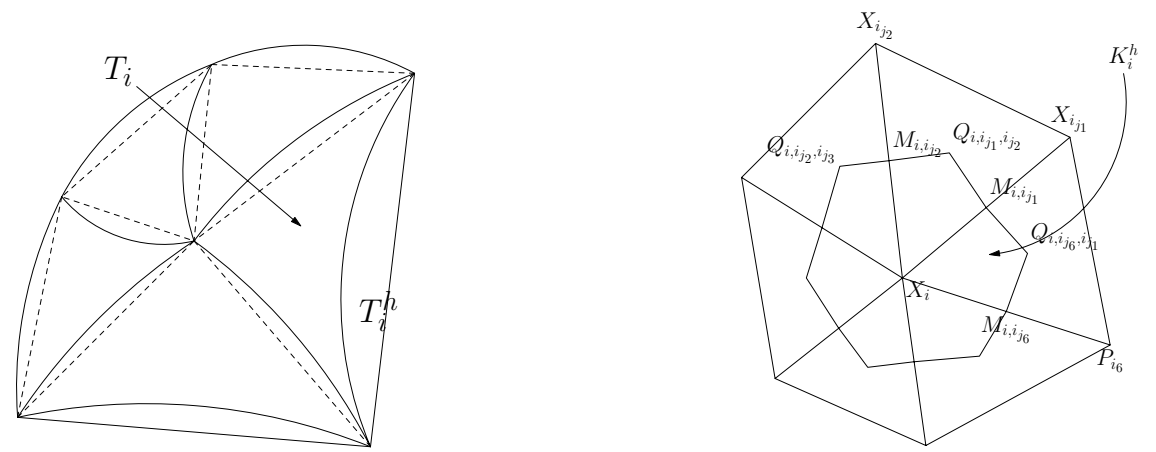

Fig. 1. Approximate mesh surface and the control volume.

where $\mathbf{p}(\mathbf{x}) \in \mathbf{S}, d(\mathbf{x})$ is the signed distance to $\mathbf{S}$, and $\overrightarrow{\mathbf{n}}(\mathbf{x})$ denotes the unit outward normal of $\mathbf{S}$ at $\mathbf{p}(\mathbf{x})$. The parameter $\delta$ can be determined by the surface curvatures (see [24]) if $\mathbf{S}$ is sufficiently smooth.

Denote by $\mathcal{T}=\left\{T_{i}\right\}_{i=1}^{m}$ the curved triangulation of the surface $\mathbf{S}$. Assume that $\mathbf{S}$ be approximated by a sequence of continuous piecewise linear complex [38] $\left\{\mathbf{S}^{h} \subset \mathbf{U}\right\}$, consisting of a sequence of regular triangulations $\left\{\mathcal{T}^{h}=\right.$ $\left.\left\{T_{i}^{h}\right\}_{i=1}^{m}\right\}$ with the mesh size approaching to zero. In order to avoid global double covering, we further assume that for each point $\mathbf{y} \in \mathbf{S}$ there is at most one point $\mathbf{x} \in \mathbf{S}^{h}$ such that $\mathbf{p}(\mathbf{x})=\mathbf{y}$, as suggested in [24]. Each $T^{h}$ contains vertices $\left\{\mathbf{x}_{i}\right\}_{i=1}^{n}$ on $\mathbf{S}$ (i.e., $\left\{\mathbf{x}_{i}\right\}_{i=1}^{n} \subset \mathbf{S} \cap \mathbf{S}^{h}$ ), see Fig. 1(left). Clearly, $\mathbf{S}^{h}$ is globally of class $C^{0,1}$. We use $m(\cdot)$ to denote the area for planar regions or the length for arcs and segments. Let $h_{i}$ denote the size of a triangle $T_{i}^{h} \in \mathcal{T}^{h}$ and define $h=\max _{i} h_{i}$ to be the mesh size for $\mathcal{T}^{h}$. We say that $\mathcal{T}^{h}$ is shape-regular if for any $T_{i}^{h} \in \mathcal{T}^{h}$

$$
c_{1} h^{2} \leq m\left(T_{i}^{h}\right) \leq c_{2} h^{2},
$$

where $c_{1}$ and $c_{2}$ are positive constants independent of $h$. By the uniqueness of the decomposition discussed above, we define $T_{i}=\left\{\mathbf{p}(\mathbf{x}) \in \mathbf{S} \mid \mathbf{x} \in T_{i}^{h}\right\}$, and let $\mathcal{T}=\left\{T_{i}\right\}_{i=1}^{m}$, then $\mathbf{S}=\cup_{i=1}^{n} T_{i}$. Note that this requires in particular that $\mathbf{p}\left(\partial \mathbf{S}^{h}\right)=\partial \mathbf{S}$.

Let the tangential gradient operator $\nabla_{s_{h}}$ on $\mathbf{S}^{h}$ be given by:

$$
\nabla_{s_{h}}=\left(\nabla_{s_{h}, 1}, \nabla_{s_{h}, 2}, \nabla_{s_{h}, 3}\right)=\nabla-\overrightarrow{\mathbf{n}}_{h}\left(\overrightarrow{\mathbf{n}}_{h} \cdot \nabla\right)
$$

where $\overrightarrow{\mathbf{n}}_{h}(\mathbf{x})=\left(n_{h 1}(\mathbf{x}), n_{h 2}(\mathbf{x}), n_{h 3}(\mathbf{x})\right)$ is the unit outward normal to $\mathbf{S}^{h}$. Since $\overrightarrow{\mathbf{n}}_{h}$ is constant on each triangle $T_{i}^{h}, \nabla_{s_{h}}$ only needs to be locally defined as a two dimensional gradient operator on the plane formed by $T_{i}^{h}$, and the Sobolev space $W^{m, p}\left(\mathbf{S}^{h}\right)$ is well-defined for $m \leq 1$.

Denote by $\mathcal{U}$ the space of continuous piecewise linear polynomials on $\mathbf{S}^{h}$ with respect to $\mathcal{T}^{h}$, that is,

$$
\mathcal{U}=\left\{U^{h} \in C^{0}\left(\mathbf{S}^{h}\right) \quad\left|\quad U^{h}\right|_{\partial \mathbf{S}^{h}}=0,\left.\quad U^{h}\right|_{T_{i}^{h}} \in \mathbb{P}_{1}\left(T_{i}^{h}\right)\right\}
$$


where $\mathbb{P}_{k}(D)$ denote the space of polynomials of degree no larger than $k$ on the planar domain $D$.

It is easy to see that $\mathcal{U} \subset H^{1}\left(\mathbf{S}^{h}\right)$ and for $U^{h} \in \mathcal{U}$ we have that $\nabla_{s_{h}} U^{h}$ is constant on each triangle $T_{i}^{h} \in \mathcal{T}^{h}$. A dual tessellation of $\mathcal{T}^{h}$ on $\mathbf{S}^{h}$ can be defined as shown in Fig. 1 (right). For each interior vertex $\mathbf{x}_{i}$, let $\chi_{i}=\left\{i_{s}\right\}_{s=1}^{m_{i}}$ be the set of indices of its neighbors, $Q_{i, i_{j}, i_{j+1}}$ (where $i_{s+1}=i_{1}$ if $s=m_{i}$ ) be the centroid of the triangle $\triangle \mathbf{x}_{i} \mathbf{x}_{i_{j}} \mathbf{x}_{i_{j+1}}$ and $M_{i, i_{j}}$ be the midpoint of $\overline{\mathbf{x}_{i} \mathbf{x}_{i_{j}}}$ for $i_{j} \in \chi_{i}$. Let $K_{i}^{h}=\cup_{i_{j} \in \chi_{i}} \Omega_{i, i_{j}, i_{j+1}}$ where $\Omega_{i, i_{j}, i_{j+1}}$ denotes the polygonal region bounded by $\mathbf{x}_{i}, M_{i, i_{j}}, Q_{i, i_{j}, i_{j+1}}$ and $M_{i, i_{j+1}}$. In general, $K_{i}^{h}$ is only piecewise planar and we define its projection onto $\mathbf{S}$ by $K_{i}=\left\{\mathbf{p}(\mathbf{x}) \in \mathbf{S} \mid \mathbf{x} \in K_{i}^{h}\right\}$. Let $\sigma$ denote the set of indices of all interior vertices of $\mathcal{T}^{h}$, then, $\mathcal{K}=\left\{K_{i}\right\}_{i \in \sigma}$ and $\mathcal{K}^{h}=\left\{K_{i}^{h}\right\}_{i \in \sigma}$ may be viewed as dual tessellations of $\mathbf{S}=\cup_{i=1}^{m} T_{i}$ and $\mathbf{S}^{h}=\cup_{i=1}^{m} T_{i}^{h}$, respectively. In the remaining part of this paper, for simplicity, we denote $i_{(j-1) \bmod \left(m_{i}\right)+1}$ by $i_{j}$, if $j>m_{i}$ and $i_{j} \in \chi_{i}\left(\mathbf{x}_{i_{j}}\right.$ is a neighbor vertex of $\mathbf{x}_{i}$ ), otherwise denote $i_{(j-1) \bmod (3)+1}$ by $i_{j}$, if $j>3$ and $\mathbf{x}_{i_{j}}$ is a vertex of $T_{i}^{h}=\triangle \mathbf{x}_{i_{1}} \mathbf{x}_{i_{2}} \mathbf{x}_{i_{3}}$.

Denote by $\mathcal{V}$ the space of grid functions on $\mathbf{S}^{h}$ with respect to $\mathcal{K}^{h}$ :

$$
\mathcal{V}=\left\{V^{h}\left|V^{h}\right|_{K_{i}^{h}} \in \mathbb{P}_{0}\left(K_{i}^{h}\right)\right\}
$$

A set of basis functions $\left\{\Psi_{i}^{h}\right\}_{i \in \sigma}$ of $\mathcal{V}$ is given by

$$
\Psi_{i}^{h}(\mathbf{x})= \begin{cases}1 & \mathbf{x} \in K_{i}^{h} \\ 0, & \mathbf{x} \in \mathbf{S}^{h}-K_{i}^{h}\end{cases}
$$

\subsection{Generalized central scheme}

We may uniquely extend a function $\phi$ defined on $\mathbf{S}$ to $\mathbf{U}$ by

$$
\phi^{l}(\mathbf{x})=\phi(\mathbf{p}(\mathbf{x})), \quad \forall \mathbf{x} \in \mathbf{U}
$$

Let $\mathbf{P}=\mathbf{I}-\overrightarrow{\mathbf{n}} \otimes \overrightarrow{\mathbf{n}}$ where $\otimes$ is the outer product defined as $\vec{a} \otimes \vec{b}=\vec{a} \vec{b}^{T}$, and it follows that

$$
\nabla_{s} \phi=\nabla \phi^{l}-\overrightarrow{\mathbf{n}}\left(\overrightarrow{\mathbf{n}} \cdot \nabla \phi^{l}\right)=\mathbf{P} \nabla \phi^{l}
$$

due to (11).

We then do the similar extension from $\mathbf{S}^{h}$ to $\mathbf{U}$. Given a function $\phi_{h}$ defined on $\mathbf{S}^{h}$, first project it onto $\mathbf{S}$ by $\phi_{h}(\mathbf{y})=\tilde{\phi}_{h}(\mathbf{p}(\mathbf{y}))$ for $\mathbf{y} \in \mathbf{S}^{h}$, then we apply (11) again to extend $\tilde{\phi}_{h}$ to $\mathbf{U}$, i.e.,

$$
\phi_{h}^{l}(\mathbf{x})=\tilde{\phi}_{h}(\mathbf{p}(\mathbf{x})), \quad \forall \mathbf{x} \in \mathbf{U}
$$


Then we successfully extend $\phi_{h}$ defined on $\mathbf{S}^{h}$ to $\mathbf{U}$ in two steps. Since all extensions of functions to $\mathbf{U}$ are constant along normals to $\mathbf{S}$, extensions of functions defined on $\mathbf{S}$ and of functions defined on $\mathbf{S}^{h}$ have the same properties.

With the above preparations, a generalized central finite volume scheme for the above steady diffusion-convection-reaction equation (1) can be defined as follows: find $u_{h} \in \mathcal{U}$ such that

$$
\mathcal{A}_{G}^{h}\left(u_{h}, \phi_{h}\right)=\left(f^{l}, \phi_{h}\right)_{s_{h}} \quad \forall \phi_{h} \in \mathcal{V}
$$

where

$$
\mathcal{A}_{G}^{h}\left(u_{h}, \phi_{h}\right)=\sum_{i \in \sigma} \phi_{h, i} \mathcal{A}_{G}^{h}\left(u_{h}, \Psi_{i}^{h}\right)
$$

and

$$
\begin{aligned}
\phi_{h, i} & =\phi_{h}\left(\mathbf{x}_{i}\right) \\
\mathcal{A}_{G}^{h}\left(u_{h}, \Psi_{i}^{h}\right) & =\int_{\partial K_{i}^{h}}\left(-a^{l} \nabla_{s_{h}} u_{h}+u_{h} \overrightarrow{\mathbf{v}}^{l}\right) \cdot \overrightarrow{\mathbf{n}}_{K_{i}^{h}} d \gamma_{h}+\int_{K_{i}^{h}} b^{l} u_{h} d s_{h} .
\end{aligned}
$$

The corresponding lifting $u_{h}^{l}$ constrained on $\mathbf{S}$ then can be regarded as the approximate solution of the model problem (1). For the existence of the approximate solution $u_{h}$ of (14) and relevant a priori error estimates, see [19] for details. Specially, for the convection-dominated case, in order to eliminate non-physical oscillations, an up-wind finite volume scheme was given in [19]. In this paper, we will focus our discussion on the generalized central scheme.

\section{A residual-based a posteriori error estimator}

Before deriving local a posteriori error estimates for the finite volume discretization (14), let us present some properties of lifts and extensions of functions defined above.

For $\mathbf{x} \in \mathbf{S}^{h}$, define

$$
\mathbf{P}_{h}(\mathbf{x})=\mathbf{I}-\overrightarrow{\mathbf{n}}_{h}(\mathbf{x}) \otimes \overrightarrow{\mathbf{n}}_{h}(\mathbf{x})
$$

and then for a function $\phi$ defined on $\mathbf{U}$, we have

$$
\nabla_{s_{h}} \phi(\mathbf{x})=\mathbf{P}_{h} \nabla \phi(\mathbf{x}), \quad \forall \mathbf{x} \in \mathbf{S}^{h}
$$

According to (8) and (13), for $\phi_{h}$ defined on $\mathbf{S}^{h}$, it holds that

$$
\nabla \phi_{h}^{l}(\mathbf{x})=(\mathbf{P}-d \mathbf{H}) \nabla \phi_{h}^{l}(\mathbf{p}(\mathbf{x})), \quad \forall \mathbf{x} \in \mathbf{S}^{h},
$$

where $\mathbf{H}: \mathbb{R}^{3} \rightarrow \mathbb{R}^{3}$ denotes the Weingarten map. Detailed discussions about $\mathbf{H}$ can be found in [15]. Since $\overrightarrow{\mathbf{n}}^{T} \cdot \overrightarrow{\mathbf{n}} \equiv 1$, it holds $\overrightarrow{\mathbf{n}} \mathbf{H}=\mathbf{H} \overrightarrow{\mathbf{n}}=0$ and $\mathbf{P H}=\mathbf{H P}=\mathbf{H}$ [15]. 
Then, for any $\mathbf{x} \in \mathbf{S}^{h}$, we obtain that

$$
\nabla \phi_{h}^{l}(\mathbf{x})=(\mathbf{I}-d \mathbf{H}) \mathbf{P} \nabla \phi_{h}^{l}(\mathbf{p}(\mathbf{x}))=(\mathbf{I}-d \mathbf{H}) \nabla_{s} \phi_{h}^{l}(\mathbf{p}(\mathbf{x})) .
$$

Combination of (16) and (18) gives us

$$
\nabla_{s_{h}} \phi_{h}(\mathbf{x})=\nabla_{s_{h}} \phi_{h}^{l}(\mathbf{x})=\mathbf{P}_{h}(\mathbf{I}-d \mathbf{H}) \mathbf{P} \nabla_{s} \phi_{h}^{l}(\mathbf{p}(\mathbf{x})) .
$$

Correspondingly, for $\psi \in H^{1}(\mathbf{S})$, we get

$$
\nabla_{s_{h}} \psi^{l}(\mathbf{x})=\mathbf{P}_{h}(\mathbf{I}-d \mathbf{H}) \mathbf{P} \nabla_{s} \psi(\mathbf{p}(\mathbf{x})), \quad \forall \mathbf{x} \in \mathbf{U} .
$$

If $\mathbf{x} \in \mathbf{S}^{h}$, (18) yields

$$
\nabla_{s} \phi_{h}^{l}(\mathbf{p}(\mathbf{x}))=(\mathbf{I}-d \mathbf{H})^{-1} \nabla \phi_{h}^{l}(\mathbf{x}),
$$

We note that the invertibility of $\mathbf{I}-d \mathbf{H}$ was proved in [15]. Next, we aim to deduce $\nabla_{s} \phi_{h}^{l}$ for given $\phi_{h} \in \mathbf{S}^{h}$. Using (19) and (20), we have

$$
\nabla_{s_{h}} \phi_{h}(\mathbf{x})=\mathbf{P}_{h} \nabla \phi_{h}^{l}(\mathbf{x}), \quad \forall \mathbf{x} \in \mathbf{S}^{h} .
$$

It is easy to see that for $\mathbf{x} \in \mathbf{S}^{h}$

$$
0=\nabla \phi_{h}^{l}(\mathbf{x}) \cdot \overrightarrow{\mathbf{n}}=\nabla_{s_{h}} \phi_{h}(\mathbf{x}) \cdot \overrightarrow{\mathbf{n}}+\left(\overrightarrow{\mathbf{n}}_{h} \cdot \overrightarrow{\mathbf{n}}\right) \nabla \phi_{h}^{l}(\mathbf{x}) \cdot \overrightarrow{\mathbf{n}}_{h},
$$

then it follows that

$$
\nabla \phi_{h}^{l}(\mathbf{x}) \cdot \overrightarrow{\mathbf{n}}_{h}=-\frac{\nabla_{s_{h}} \phi_{h}(\mathbf{x}) \cdot \overrightarrow{\mathbf{n}}}{\overrightarrow{\mathbf{n}}_{h} \cdot \overrightarrow{\mathbf{n}}}
$$

Thus we have

and

$$
\nabla \phi_{h}^{l}(\mathbf{x})=\left(\mathbf{I}-\frac{\overrightarrow{\mathbf{n}}_{h} \otimes \overrightarrow{\mathbf{n}}}{\overrightarrow{\mathbf{n}}_{h} \cdot \overrightarrow{\mathbf{n}}}\right) \nabla_{s_{h}} \phi_{h}(\mathbf{x})
$$

$$
\nabla_{s} \phi_{h}^{l}(\mathbf{p}(\mathbf{x}))=(\mathbf{I}-d \mathbf{H})^{-1}\left(\mathbf{I}-\frac{\overrightarrow{\mathbf{n}}_{h} \otimes \overrightarrow{\mathbf{n}}}{\overrightarrow{\mathbf{n}}_{h} \cdot \overrightarrow{\mathbf{n}}}\right) \nabla_{s_{h}} \phi_{h}(\mathbf{x})
$$

Define

$$
\mu_{h}(\mathbf{x})=\frac{d s(\mathbf{x})}{d s_{h}(p(\mathbf{x}))}, \quad \xi_{h}(\mathbf{x})=\frac{d \gamma(\mathbf{x}))}{d \gamma_{h}(p(\mathbf{x}))}
$$

for any $\mathbf{x} \in \mathbf{S}^{h}$. Since $\mathbf{S}$ and $\partial \mathbf{S}$ are sufficiently smooth, we have (see $\left.[19,22]\right)$

$$
\left|1-\mu_{h}(\mathbf{x})\right| \leq c h^{2}, \quad\left|1-\xi_{h}(\mathbf{x})\right| \leq c h^{2} .
$$

Finally, we cite the following results from [22] for later use:

Lemma 3 For any $\phi \in H^{1}(\mathbf{S})$, there exist some generic constants $c_{1}, c_{2}, c_{3}, c_{4}>$ 0 such that

$$
\left\{\begin{array}{l}
c_{1}\left\|\phi^{l}\right\|_{L^{2}\left(T_{i}^{h}\right)} \leq\|\phi\|_{L^{2}\left(T_{i}\right)} \leq c_{2}\left\|\phi^{l}\right\|_{L^{2}\left(T_{i}^{h}\right)}, \\
c_{3}\left\|\phi^{l}\right\|_{H^{1}\left(T_{i}^{h}\right)} \leq\|\phi\|_{H^{1}\left(T_{i}\right)} \leq c_{4}\left\|\phi^{l}\right\|_{H^{1}\left(T_{i}^{h}\right)}
\end{array}\right.
$$


for any $T_{i} \in \mathcal{T}$.

With all the above notations, we have

$$
\int_{\mathbf{S}^{h}} \nabla_{s_{h}} \phi_{h} \cdot \nabla_{s_{h}} \psi_{h} d s_{h}=\int_{\mathbf{S}} \mathbf{A}_{h}^{l} \nabla_{s} \phi_{h}^{l} \cdot \nabla_{s} \psi_{h}^{l} d s,
$$

where $\mathbf{A}_{h}^{l}(\mathbf{p}(\mathbf{x}))=\mathbf{A}_{h}(\mathbf{x})=\left(\frac{1}{\mu_{h}} \mathbf{P}(\mathbf{I}-d \mathbf{H}) \mathbf{P}_{h}(\mathbf{I}-d \mathbf{H}) \mathbf{P}\right)(\mathbf{x}), \quad \forall \mathbf{x} \in \mathbf{S}^{h}$.

Similarly, it holds that

$$
\int_{\mathbf{S}^{h}} \nabla_{s_{h}} \phi_{h} \psi_{h} d s_{h}=\int_{\mathbf{S}} \mathbf{B}_{h}^{l} \nabla_{s} \phi_{h}^{l} \psi_{h}^{l} d s
$$

where $\mathbf{B}_{h}^{l}(\mathbf{p}(\mathbf{x}))=\mathbf{B}_{h}(\mathbf{x})=\left(\frac{1}{\mu_{h}} \mathbf{P}_{h}(\mathbf{I}-d \mathbf{H}) \mathbf{P}\right)(\mathbf{x}), \quad \forall \mathbf{x} \in \mathbf{S}^{h}$.

\subsection{An a posteriori estimator and its reliability}

In the following, we will derive an energy-type (or $H^{1}$-type) a posteriori estimate for the discrete solution $u_{h}$, i.e., to estimate $\left\|u-u_{h}^{l}\right\|_{E}\left(\right.$ or $\left.\left\|u-u_{h}^{l}\right\|_{H^{1}(\mathbf{S})}\right)$.

Let $e$ be an edge shared by elements $T_{1}$ and $T_{2}$ which have normals $\overrightarrow{\mathbf{n}}_{T_{1}}$ and $\overrightarrow{\mathbf{n}}_{T_{2}}$ on $e$, respectively, then we can define

$$
\left[\left[a^{l} \nabla_{s_{h}} u_{h}-\overrightarrow{\mathbf{v}} u_{h}\right]\right]=\left.\left(a^{l} \nabla_{s_{h}} u_{h}-\overrightarrow{\mathbf{v}} u_{h}\right)\right|_{T_{1}} \cdot \overrightarrow{\mathbf{n}}_{T_{1}}+\left.\left(a^{l} \nabla_{s_{h}} u_{h}-\overrightarrow{\mathbf{v}} u_{h}\right)\right|_{T_{2}} \cdot \overrightarrow{\mathbf{n}}_{T_{2}},
$$

in particular, if $e \subset \partial \mathbf{S}_{h}$ we set $\left[\left[a^{l} \nabla_{s_{h}} u_{h}-\overrightarrow{\mathbf{v}} u_{h}\right]\right] \equiv 0$. By Green's formula, it 
follows that for any $\phi \in H^{1}(\mathbf{S})$ :

$$
\begin{aligned}
\mathcal{A}\left(u-u_{h}^{l}, \phi\right)= & \int_{\mathbf{S}} f \phi d s-\mathcal{A}\left(u_{h}^{l}, \phi\right) \\
= & \int_{\mathbf{S}^{h}} f^{l} \mu_{h} \phi^{l} d s_{h}-\int_{\mathbf{S}} a \nabla_{s} u_{h}^{l} \cdot \nabla_{s} \phi d s+\int_{\mathbf{S}}\left(\overrightarrow{\mathbf{v}} \cdot \nabla_{s} \phi\right) u_{h}^{l} d s-\int_{\mathbf{S}} b u_{h}^{l} \phi d s \\
= & \int_{\mathbf{S}^{h}} f^{l} \mu_{h} \phi^{l} d s_{h}-\int_{\mathbf{S}} a\left(\mathbf{P}-\mathbf{A}_{h}^{l}\right) \nabla_{s} u_{h}^{l} \cdot \nabla_{s} \phi d s+\int_{\mathbf{S}}\left(\mathbf{I}-\mathbf{B}_{h}^{l}\right)\left(\overrightarrow{\mathbf{v}} \cdot \nabla_{s} \phi\right) u_{h}^{l} d s \\
& \quad-\int_{\mathbf{S}^{h}} a^{l} \nabla_{s_{h}} u_{h} \cdot \nabla_{s_{h}} \phi^{l} d s_{h}+\int_{\mathbf{S}^{h}}\left(\overrightarrow{\mathbf{v}}^{l} \cdot \nabla_{s_{h}} \phi^{l}\right) u_{h} d s_{h}-\int_{\mathbf{S}} b u_{h}^{l} \phi d s \\
= & \int_{\mathbf{S}^{h}} f^{l} \mu_{h} \phi^{l} d s_{h}-\int_{\mathbf{S}} a\left(\mathbf{P}-\mathbf{A}_{h}^{l}\right) \nabla_{s} u_{h}^{l} \cdot \nabla_{s} \phi d s+\int_{\mathbf{S}}\left(\mathbf{I}-\mathbf{B}_{h}^{l}\right)\left(\overrightarrow{\mathbf{v}} \cdot \nabla_{s} \phi\right) u_{h}^{l} d s \\
& +\int_{\mathbf{S}^{h}} \nabla_{s_{h}}\left(a^{l} \nabla_{s_{h}} u_{h}\right) \phi^{l} d s_{h}-\sum_{T_{i}^{h} \in \mathcal{T}^{h}} \int_{\partial T_{i}^{h}} a^{l}\left(\nabla_{s_{h}} u_{h} \cdot \overrightarrow{\mathbf{n}}_{T_{i}^{h}}\right) \phi^{l} d \gamma_{h} \\
& -\int_{\mathbf{S}^{h}} \nabla_{s_{h}}\left(\overrightarrow{\mathbf{v}}^{l} u_{h}\right) \cdot \phi^{l} d s_{h}+\sum_{T_{i}^{h} \in \mathcal{T}^{h}} \int_{\partial T_{i}^{h}} u_{h} \overrightarrow{\mathbf{v}}^{l} \cdot \overrightarrow{\mathbf{n}}_{T_{i}^{h}} \cdot \phi^{l} d \gamma_{h}-\int_{\mathbf{S}} b u_{h}^{l} \phi d s . \\
= & \int_{S_{h}} f^{l} \mu_{h} \phi^{l} d s_{h}-\int_{S} a\left(\mathbf{P}-\mathbf{A}_{h}^{l}\right) \nabla_{s} u_{h}^{l} \nabla_{s} \phi d s+\int_{S}\left(\mathbf{I}-\mathbf{B}_{h}^{l}\right)\left(\overrightarrow{\mathbf{v}} \cdot \nabla_{s} \phi\right) u_{h}^{l} d s \\
& +\int_{\mathbf{S}^{h}} \nabla_{s_{h}}\left(a^{l} \nabla_{s_{h}} u_{h}\right) \phi^{l} d s_{h}-\int_{\mathbf{S}^{h}} \nabla_{s_{h}}\left(\overrightarrow{\mathbf{v}}^{l} u_{h}\right) \cdot \phi^{l} d s_{h} \\
& \quad-\frac{1}{2} \sum_{T_{i}^{h} \in \mathcal{T}} \int_{\partial T_{i}^{h}}\left[\left[a^{l} \nabla_{s_{h}} u_{h}-\overrightarrow{\mathbf{v}} u_{h}\right]\right] \phi^{l} d \gamma_{h}-\int_{S} b u_{h}^{l} \phi d s . \quad(24)
\end{aligned}
$$

On the other hand, for any $u \in C^{0}\left(\mathbf{S}^{h}\right)$, denote by $\Pi(u)$ the interpolation of $u$ onto $\mathcal{V}$, i.e. $\Pi(u) \in \mathcal{V}$ and $\Pi(u)\left(\mathbf{x}_{i}\right)=u\left(\mathbf{x}_{i}\right)$ for all $i \in \sigma$. Then it holds that

$$
\begin{aligned}
-\sum_{i \in \sigma} \phi_{i} \int_{\partial K_{i}^{h}} a^{l} \nabla_{s_{h}} u_{h} \cdot \overrightarrow{\mathbf{n}}_{K^{h}} d \gamma_{h} \\
=\sum_{T_{i}^{h} \in \mathcal{T}^{h}}\left(-\sum_{j=1}^{3} \int_{\partial K_{i j}^{h} \cap T_{i}^{h}}\left(a^{l} \nabla_{s_{h}} u_{h} \cdot \overrightarrow{\mathbf{n}}_{K_{i j}^{h}}\right) \Pi\left(\phi^{l}\right) d \gamma_{h}\right) \\
=\sum_{T_{i}^{h} \in \mathcal{T}^{h}}\left(-\int_{T_{i}^{h}} \nabla_{s_{h}} \cdot\left(a^{l} \nabla_{s_{h}} u_{h}\right) \Pi\left(\phi^{l}\right) d s_{h}+\int_{\partial T_{i}^{h}}\left(a^{l} \nabla_{s_{h}} u_{h} \cdot \overrightarrow{\mathbf{n}}_{T_{i}^{h}}\right) \Pi\left(\phi^{l}\right) d \gamma_{h}\right)
\end{aligned}
$$

and similarly

$$
\begin{aligned}
\sum_{i \in \sigma} \phi_{i} & \int_{\partial K_{i}^{h}} u_{h} \overrightarrow{\mathbf{v}}^{l} \cdot \overrightarrow{\mathbf{n}}_{K_{i}^{h}} d \gamma_{h} \\
& =\sum_{T_{i}^{h} \in \mathcal{T}^{h}}\left(-\int_{T_{i}^{h}} \nabla_{s_{h}} \cdot\left(u_{h} \overrightarrow{\mathbf{v}}^{l}\right) \Pi\left(\phi^{l}\right) d s_{h}+\int_{\partial T_{i}^{h}}\left(u_{h} \overrightarrow{\mathbf{v}}^{l} \cdot \overrightarrow{\mathbf{n}}_{T_{i}^{h}}\right) \Pi\left(\phi^{l}\right) d \gamma_{h}\right) .
\end{aligned}
$$


Thus,

$$
\begin{aligned}
& \mathcal{A}_{G}^{h}\left(u_{h}, \Pi\left(\phi^{l}\right)\right) \\
& =-\sum_{i \in \sigma} \phi_{i} \int_{\partial K_{i}^{h}} a^{l} \nabla_{s_{h}} u_{h} \cdot \overrightarrow{\mathbf{n}}_{K_{i}^{h}} d \gamma_{h}+\sum_{i \in \sigma} \phi_{i} \int_{\partial K_{i}^{h}} u_{h} \overrightarrow{\mathbf{v}}^{l} \cdot \overrightarrow{\mathbf{n}}_{K_{i}^{h}} d \gamma_{h}+\int_{\mathbf{S}_{h}} b^{l} u_{h} \Pi\left(\phi^{l}\right) d s_{h} \\
& =-\int_{\mathbf{S}_{h}} \nabla_{s_{h}} \cdot\left(a^{l} \nabla_{s_{h}} u_{h}\right) \Pi\left(\phi^{l}\right) d s_{h}+\int_{\mathbf{S}_{h}} \nabla_{s_{h}} \cdot\left(\overrightarrow{\mathbf{v}}^{l} u_{h}\right) \Pi\left(\phi^{l}\right) d s_{h}+\int_{\mathbf{S}_{h}} b^{l} u_{h} \Pi\left(\phi^{l}\right) d s_{h} \\
& \quad+\frac{1}{2} \sum_{T_{i}^{h} \in \mathcal{T}^{h}} \int_{\partial T_{i}^{h}}\left[\left[a^{l} \nabla_{s_{h}} u_{h}-\overrightarrow{\mathbf{v}}^{l} u_{h}\right]\right] \phi^{l} d \gamma_{h} .
\end{aligned}
$$

Applying the equalities (24)-(27), we obtain

$$
\begin{aligned}
\mathcal{A}\left(u-u_{h}^{l}, \phi\right) & =\mathcal{A}\left(u-u_{h}^{l}, \phi\right)+\mathcal{A}_{G}^{h}\left(u_{h}, \Pi\left(\phi^{l}\right)\right)-\mathcal{A}_{G}^{h}\left(u_{h}, \Pi\left(\phi^{l}\right)\right) \\
= & \int_{\mathbf{S}^{h}} f^{l} \mu_{h} \phi^{l} d s_{h}-\int_{\mathbf{S}} a\left(\mathbf{P}-\mathbf{A}_{h}^{l}\right) \nabla_{s} u_{h}^{l} \cdot \nabla_{s} \phi d s+\int_{\mathbf{S}}\left(\mathbf{I}-\mathbf{B}_{h}^{l}\right)\left(\overrightarrow{\mathbf{v}} \cdot \nabla_{s} \phi\right) u_{h}^{l} d s \\
& +\int_{\mathbf{S}^{h}} \nabla_{s_{h}} \cdot\left(a^{l} \nabla_{s_{h}} u_{h}\right)\left(\phi^{l}-\Pi\left(\phi^{l}\right)\right) d s_{h}-\int_{\mathbf{S}^{h}} \nabla_{s_{h}} \cdot\left(\overrightarrow{\mathbf{v}}^{l} u_{h}\right)\left(\phi^{l}-\Pi\left(\phi^{l}\right)\right) d s_{h} \\
& -\frac{1}{2} \sum_{T_{i}^{h} \in \mathcal{T}^{h}} \int_{\partial T_{i}^{h}}\left[\left[a^{l} \nabla_{s_{h}} u_{h}-u_{h} \overrightarrow{\mathbf{v}}^{l}\right]\right]\left(\phi^{l}-\Pi\left(\phi^{l}\right)\right) d \gamma_{h} \\
& -\int_{\mathbf{S}^{h}} b^{l} \mu_{h} u_{h} \phi^{l} d s_{h}+\int_{\mathbf{S}^{h}} b^{l} u_{h} \Pi\left(\phi^{l}\right) d s_{h}-\int_{\mathbf{S}^{h}} f^{l} \Pi\left(\phi^{l}\right) d s_{h} \\
= & \int_{\mathbf{S}^{h}}\left(f^{l} \mu_{h}+\nabla_{s_{h}} \cdot\left(a^{l} \nabla_{s_{h}} u_{h}-\overrightarrow{\mathbf{v}} u_{h}\right)-b^{l} \mu_{h} u_{h}\right)\left(\phi^{l}-\Pi\left(\phi^{l}\right)\right) d s_{h} \\
& -\int_{\mathbf{S}}\left(a\left(\mathbf{P}-\mathbf{A}_{h}^{l}\right) \nabla_{s} u_{h}^{l}-\left(\mathbf{I}-\mathbf{B}_{h}^{l}\right) \overrightarrow{\mathbf{v}} u_{h}^{l}\right) \cdot \nabla_{s} \phi d s \\
& -\frac{1}{2} \sum_{T_{i}^{h} \in \mathcal{T}^{h}} \int_{\partial T_{i}^{h}}\left[\left[a^{l} \nabla_{s} u_{h}-u_{h} \overrightarrow{\mathbf{v}}^{l}\right]\right]\left(\phi^{l}-\Pi\left(\phi^{l}\right)\right) d \gamma_{h} \\
& +\int_{S_{h}}\left(1-\mu_{h}\right)\left(b^{l} u_{h}-f^{l}\right) \Pi\left(\phi^{l}\right) d s_{h} \\
= & I_{1}+I_{2}+I_{3}+I_{4}
\end{aligned}
$$

where

$$
\begin{aligned}
& I_{1}=\int_{\mathbf{S}^{h}}\left(f^{l} \mu_{h}+\nabla_{s_{h}} \cdot\left(a^{l} \nabla_{s_{h}} u_{h}-\overrightarrow{\mathbf{v}} \cdot u_{h}\right)-b^{l} \mu_{h} u_{h}\right)\left(\phi^{l}-\Pi\left(\phi^{l}\right)\right) d s_{h}, \\
& I_{2}=-\frac{1}{2} \sum_{T_{i}^{h} \in \mathcal{T}^{h}} \int_{\partial T_{i}^{h}}\left[\left[a^{l} \nabla_{s} u_{h}-u_{h} \overrightarrow{\mathbf{v}}^{l}\right]\right]\left(\phi^{l}-\Pi\left(\phi^{l}\right)\right) d \gamma_{h}, \\
& I_{3}=-\int_{\mathbf{S}}\left(a\left(\mathbf{P}-\mathbf{A}_{h}^{l}\right) \nabla_{s} u_{h}^{l}-\left(\mathbf{I}-\mathbf{B}_{h}^{l}\right) \overrightarrow{\mathbf{v}} u_{h}^{l}\right) \cdot \nabla_{s} \phi d s \\
& I_{4}=\int_{\mathbf{S}_{h}}\left(1-\mu_{h}\right)\left(b^{l} u_{h}-f^{l}\right) \Pi\left(\phi^{l}\right) d s_{h} .
\end{aligned}
$$

Next, we will analyze the above four terms, respectively, to get an appropriate estimator. 
First, let us define

$$
R=f^{l} \mu_{h}+\nabla_{s_{h}} \cdot\left(a^{l} \nabla_{s_{h}} u_{h}-\overrightarrow{\mathbf{v}} u_{h}\right)-b^{l} \mu_{h} u_{h}
$$

and

$$
r=\left[\left[a^{l} \nabla_{s} u_{h}-u_{h} \overrightarrow{\mathbf{v}}^{l}\right]\right] .
$$

Then it follows that

$$
\begin{aligned}
& \left|I_{1}\right|=\left|\sum_{T_{i}^{h} \in \mathcal{T}^{h}} \int_{T_{i}^{h}} R\left(\phi^{l}-\Pi\left(\phi^{l}\right)\right) d s_{h}\right| \leq \sum_{T_{i}^{h} \in \mathcal{T}^{h}}\|R\|_{L^{2}\left(T_{i}^{h}\right)}\left\|\phi^{l}-\Pi\left(\phi^{l}\right)\right\|_{L^{2}\left(T_{i}^{h}\right)} \\
& \quad \leq c \sum_{T_{i}^{h} \in \mathcal{T}^{h}} h_{i}\|R\|_{L^{2}\left(T_{i}^{h}\right)}\|\phi\|_{H^{1}\left(T_{i}\right)}
\end{aligned}
$$

and

$$
\begin{aligned}
\left|I_{2}\right|=\mid & -\frac{1}{2} \sum_{T_{i}^{h} \in \mathcal{T}^{h}} \int_{\partial T_{i}^{h}} r\left(\phi^{l}-\Pi\left(\phi^{l}\right)\right) d \gamma_{h} \mid \leq \frac{1}{2} \sum_{T_{i}^{h} \in \mathcal{T}^{h}}\|r\|_{L^{2}\left(\partial T_{i}^{h}\right)}\left\|\phi^{l}-\Pi\left(\phi^{l}\right)\right\|_{L^{2}\left(\partial T_{i}^{h}\right)} \\
& \leq c \sum_{T_{i}^{h} \in \mathcal{T}^{h}} \frac{1}{2} h_{i}^{\frac{1}{2}}\|r\|_{L^{2}\left(\partial T_{i}^{h}\right)}\|\phi\|_{H^{1}\left(T_{i}\right)} .
\end{aligned}
$$

Using Cauchy-Schwartz inequality and the trace theorem, we immediately get

$$
\begin{aligned}
\left|I_{1}+I_{2}\right| & \leq c \sum_{T_{i}^{h} \in \mathcal{T}^{h}}\left(h_{i}\|R\|_{L^{2}\left(T_{i}^{h}\right)}+\frac{1}{2} h_{i}^{\frac{1}{2}}\|r\|_{L^{2}\left(\partial T_{i}^{h}\right)}\right)\|\phi\|_{H^{1}\left(T_{i}\right)} \\
& \leq c\left(\sum_{T_{i}^{h} \in \mathcal{T}^{h}} h_{i}^{2}\|R\|_{L^{2}\left(T_{i}^{h}\right)}^{2}+\frac{1}{4} \sum_{T_{i}^{h} \in \mathcal{T}^{h}} h_{i}\|r\|_{L^{2}\left(\partial T_{i}^{h}\right)}^{2}\right)^{\frac{1}{2}}\|\phi\|_{H^{1}(\mathbf{S})} .
\end{aligned}
$$

As for $I_{3}$, we have

$$
\begin{aligned}
\left|I_{3}\right| \leq & \sum_{T_{i}^{h} \in \mathcal{T}^{h}}\left\|a\left(\mathbf{P}-\mathbf{A}_{h}^{l}\right) \nabla_{s} u_{h}^{l}-\left(\mathbf{I}-\mathbf{B}_{h}^{l}\right) \overrightarrow{\mathbf{v}} u_{h}^{l}\right\|_{L^{2}\left(T_{i}\right)}\left\|\nabla_{s} \phi\right\|_{L^{2}\left(T_{i}\right)} \\
\leq c & \sum_{T_{i}^{h} \in \mathcal{T}^{h}} \| a \sqrt{\mu_{h}}\left(\mathbf{P}-\mathbf{A}_{h}\right)(\mathbf{I}-d \mathbf{H})^{-1}\left(\mathbf{I}-\frac{\overrightarrow{\mathbf{n}}_{h} \otimes \overrightarrow{\mathbf{n}}}{\overrightarrow{\mathbf{n}}_{h} \cdot \overrightarrow{\mathbf{n}}}\right) \nabla_{s_{h}} u_{h} \\
& -\sqrt{\mu_{h}}\left[\mathbf{I}-\mathbf{B}_{h}\right] \overrightarrow{\mathbf{v}}^{l} u_{h}\left\|_{L^{2}\left(T_{i}^{h}\right)}\right\| \nabla_{s} \phi \|_{L^{2}\left(T_{i}\right)} .
\end{aligned}
$$

Now set

$$
\mathbf{C}_{h}=a \sqrt{\mu_{h}}\left(\mathbf{P}-\mathbf{A}_{h}\right)(\mathbf{I}-d \mathbf{H})^{-1}\left(\mathbf{I}-\frac{\overrightarrow{\mathbf{n}}_{h} \otimes \overrightarrow{\mathbf{n}}}{\overrightarrow{\mathbf{n}}_{h} \cdot \overrightarrow{\mathbf{n}}}\right),
$$

then we finally arrive at

$$
\begin{aligned}
\left|I_{3}\right| & \leq c \sum_{T_{i}^{h} \in \mathcal{T}^{h}}\left\|\mathbf{C}_{h} \nabla_{s_{h}} u_{h}-\sqrt{\mu_{h}}\left(\mathbf{I}-\mathbf{B}_{h}\right) \overrightarrow{\mathbf{v}}^{l} u_{h}\right\|_{L^{2}\left(T_{i}^{h}\right)}\left\|\phi^{l}\right\|_{H^{1}\left(T_{i}^{h}\right)} \\
& \leq c \beta_{1}^{*}\left\|\mathbf{C}_{h} \nabla_{s_{h}} u_{h}-\sqrt{\mu_{h}}\left(\mathbf{I}-\mathbf{B}_{h}\right) \overrightarrow{\mathbf{v}}^{l} u_{h}\right\|_{L^{2}\left(\mathbf{S}^{h}\right)}\|\phi\|_{H^{1}(\mathbf{S})}
\end{aligned}
$$


where $\beta_{1}^{*}>0$ is a generic constant that also depends on the curvature information of the surface $\mathbf{S}$. For more discussions about this issue, see [15]. Also it holds that

$$
\begin{aligned}
\left|I_{4}\right| & =\left|\int_{S_{h}}\left(1-\mu_{h}\right)\left(b^{l} u_{h}-f^{l}\right) \Pi\left(\phi^{l}\right) d s_{h}\right| \\
& \leq \sum_{T_{i}^{h} \in \mathcal{T}}\left|\int_{T_{i}^{h}}\left(1-\mu_{h}\right)\left(b^{l} u_{h}-f^{l}\right) \Pi\left(\phi^{l}\right) d s_{h}\right| \\
& \leq \sum_{T_{i}^{h} \in \mathcal{T}}\left\|\left(1-\mu_{h}\right)\left(b^{l} u_{h}-f^{l}\right)\right\|_{L^{2}\left(T_{i}^{h}\right)}\left\|\Pi\left(\phi^{l}\right)\right\|_{L^{2}\left(T_{i}^{h}\right)} \\
& \leq c \beta_{2}^{*}\left(\sum_{T_{i}^{h} \in \mathcal{T}}\left\|\left(1-\mu_{h}\right)\left(b^{l} u_{h}-f^{l}\right)\right\|_{L^{2}\left(T_{i}^{h}\right)}^{2}\right)^{\frac{1}{2}}\|\phi\|_{H^{1}(\mathbf{S})}
\end{aligned}
$$

where $\beta_{2}^{*}>0$ is again a generic constant that also depends on the curvature information.

Thus, by letting $\phi=u-u_{h}^{l}$, we get an estimator as follows:

$$
\left\|u-u_{h}^{l}\right\|_{E} \leq c\left(\sum_{T_{i}^{h} \in \mathcal{T}^{h}} \mathbf{R}_{T_{i}^{h}, 1}^{2}+\mathbf{R}_{T_{i}^{h}, 2}^{2}+\mathbf{R}_{T_{i}^{h}, 3}^{2}\right)^{\frac{1}{2}}
$$

where

$$
\begin{aligned}
& \mathbf{R}_{T_{i}^{h}, 1}=\left(h_{i}^{2}\|R\|_{L^{2}\left(T_{i}^{h}\right)}^{2}+\frac{1}{4} h_{i}\|r\|_{L^{2}\left(\partial T_{i}^{h}\right)}^{2}\right)^{\frac{1}{2}}, \\
& \mathbf{R}_{T_{i}^{h}, 2}=\sqrt{\beta_{1}^{*}}\left\|\mathbf{C}_{h} \nabla_{s_{h}} u_{h}-\sqrt{\mu_{h}}\left(\mathbf{I}-\mathbf{B}_{h}\right) \overrightarrow{\mathbf{v}}^{l} \cdot u_{h}\right\|_{L^{2}\left(T_{i}^{h}\right)}, \\
& \mathbf{R}_{T_{i}^{h}, 3}=\sqrt{\beta_{2}^{*}}\left\|\left(1-\mu_{h}\right)\left(b^{l} u_{h}-f^{l}\right)\right\|_{L^{2}\left(T_{i}^{h}\right)} \text {. }
\end{aligned}
$$

Now let us define the local explicit a posteriori error estimator $\eta_{T_{i}^{h}}$ on each triangle $T_{i}^{h} \in \mathcal{T}^{h}$ by

$$
\eta_{T_{i}^{h}}^{2}=\mathbf{R}_{T_{i}^{h}, 1}^{2}+\mathbf{R}_{T_{i}^{h}, 2}^{2}+\mathbf{R}_{T_{i}^{h}, 3}^{2}
$$

and the following result is naturally obtained:

Theorem 4 (Reliability of $\eta_{T_{i}^{h}}$ ) Assume that $u \in H_{0}^{1}(\mathbf{S})$ is the weak solution of the problem (6), and $u^{h} \in \mathcal{U}$ is the solution of the discrete problem (14). Then under Assumption 1, there exists a generic constant $c>0$ such that

$$
\left\|u-u_{h}^{l}\right\|_{E} \leq c \eta_{\mathcal{T}^{h}}
$$

where $\eta_{\mathcal{T}^{h}}=\left(\sum_{T_{i}^{h} \in \mathcal{T}^{h}} \eta_{T_{i}^{h}}^{2}\right)^{1 / 2}$.

Remark 5 For any curved triangle $T_{i} \in \mathcal{T}$, one observes that

$$
\left|1-\mu_{h}(\mathbf{x})\right| \leq c h_{i}^{2}, \quad|d(\mathbf{x})| \leq c h_{i}^{2}, \quad\left\|\left(\mathbf{P}-\mathbf{A}_{h}\right)(\mathbf{x})\right\|_{l^{2} \rightarrow l^{2}} \leq c h_{i}^{2}
$$


for any $\mathbf{x} \in T_{i}$, then the following inequalities can be easily obtained

$$
\begin{gathered}
\left\|\mathbf{C}_{h}\right\|_{l^{2} \rightarrow l^{2}} \leq c_{1}\left\|\mathbf{P}-\mathbf{A}_{h}\right\|_{l^{2} \rightarrow l^{2}} \leq c_{2} h_{i}^{2}, \\
\left\|\mathbf{I}-\mathbf{B}_{h}\right\|_{l^{2} \rightarrow l^{2}} \leq c_{3} h_{i}^{2} .
\end{gathered}
$$

Thus we know that the last two terms in (40) are of higher order compared with the first one.

\subsection{Efficiency of the a posteriori estimator}

In this section, we aim to prove the efficiency of the estimator derived in the previous section, i.e., $\eta_{T_{i}^{h}}$ does not overestimate the true error.

Theorem 6 Assume that $u \in H_{0}^{1}(\mathbf{S})$ is the solution of the problem (6), and $u^{h} \in \mathcal{U}$ is the solution of the discrete problem (14). We also assume that $\mathcal{T}$ is shape-regular. Then under Assumption 1, it holds that for any $T_{i}^{h} \in \mathcal{T}^{h}$,

$$
\begin{gathered}
\eta_{T_{i}^{h}} \leq c\left[\left\|\mathbf{A}_{h}\right\|_{L^{\infty}\left(T^{h}\right)}^{1 / 2}\left(\left\|u-u_{h}^{l}\right\|_{H^{1}\left(T_{i}\right)}+\left\|\mathbf{C}_{h} \nabla_{s_{h}} u_{h}\right\|_{L^{2}\left(T_{i}^{h}\right)}+\left\|\sqrt{\mu_{h}}(\mathbf{I}-\mathbf{B}) \overrightarrow{\mathbf{v}}^{l} u_{h}\right\|_{L^{2}\left(T_{i}^{h}\right)}\right)\right. \\
\left.+h_{i}\|R-\bar{R}\|_{L^{2}\left(T_{i}^{h}\right)}+h_{i}^{1 / 2}\|r-\bar{r}\|_{L^{2}\left(\partial T_{i}^{h}\right)}\right]
\end{gathered}
$$

where $c$ is a generic constant, and $\bar{R}$ and $\bar{r}$ are piecewise linear approximation of $R$ and $r$, with respect to the triangulation $\mathcal{T}^{h}$.

Proof: The proof will follow the well-known framework by Verfürth [39]. First, we aim to bound $\|R\|_{L^{2}\left(T_{i}^{h}\right)}$. Define the bubble function [39] $\phi_{T_{i}^{h}}$ on $T_{i}^{h}=$ $\triangle x_{i_{1}} x_{i_{2}} x_{i_{3}}$ by $\phi_{T_{i}^{h}}=\prod_{j=1}^{3} \zeta_{x_{i_{j}}}$, where each $\zeta_{x_{i_{j}}} \in \mathcal{U}$ and $\zeta_{x_{i}}\left(x_{j}\right)=\delta_{i, j}$, such that $\left.\phi_{T_{i}^{h}}\right|_{\partial T_{i}^{h}}=0$ and $\phi_{T_{i}^{h}}=0$ outside $T^{h}$. Set $\bar{R} \in \mathcal{U}$ be a piecewise linear approximation to $R$ on $T_{i}^{h}$. Let us take $\psi=\bar{R} \phi_{T_{i}^{h}}$ in (24), and apply Poincare inequality and Theorem 2.2 in [2], then we obtain

$$
\begin{aligned}
\int_{T_{i}^{h}} R \bar{R} \phi_{T_{i}^{h}} d s_{h}= & \int_{T_{i}} a \nabla_{s}\left(u-u_{h}^{l}\right) \cdot \nabla_{s}\left(\bar{R}^{l} \phi_{T_{i}^{h}}^{l}\right) d s-\int_{T_{i}}\left(u-u_{h}^{l}\right) \overrightarrow{\mathbf{v}} \cdot \nabla_{s}\left(\bar{R}^{l} \phi_{T_{i}^{h}}^{l}\right) d s \\
& +\int_{T_{i}} b\left(u-u_{h}^{l}\right) \bar{R}^{l} \phi_{T_{i}^{h}}^{l} d s+\int_{T_{i}} a\left(\mathbf{P}-\mathbf{A}_{h}^{l}\right) \nabla_{s} u_{h}^{l} \cdot \nabla_{s}\left(\bar{R}^{l} \phi_{T_{i}^{h}}^{l}\right) d s \\
& -\int_{T_{i}}\left(\mathbf{I}-\mathbf{B}_{h}^{l}\right) \overrightarrow{\mathbf{v}} u_{h}^{l} \cdot \nabla_{s}\left(\bar{R}^{l} \phi_{T_{i}^{h}}^{l}\right) d s
\end{aligned}
$$


and then

$$
\begin{gathered}
|| \int_{T_{i}^{h}} R \bar{R} \phi_{T_{i}^{h}} d s_{h} \mid \\
\leq\left(\left\|u-u_{h}^{l}\right\|_{H^{1}\left(T_{i}\right)}+\left\|a\left(\mathbf{P}-\mathbf{A}_{h}^{l}\right) \nabla_{s} u_{h}^{l}\right\|_{L^{2}\left(T_{i}\right)}+\left\|\left(\mathbf{I}-\mathbf{B}_{h}^{l}\right) \overrightarrow{\mathbf{v}} u_{h}^{l}\right\|_{L^{2}\left(T_{i}\right)}\right) \\
\cdot\left\|\nabla_{s}\left(\bar{R}^{l} \phi_{T_{i}^{h}}^{l}\right)\right\|_{L^{2}\left(T_{i}\right)} \\
\leq\left(\left\|u-u_{h}^{l}\right\|_{H^{1}\left(T_{i}\right)}+\left\|\mathbf{C}_{h} \nabla_{s_{h}} u_{h}\right\|_{L^{2}\left(T_{i}^{h}\right)}+\left\|\sqrt{\mu_{h}}\left[\mathbf{I}-\mathbf{B}_{h}\right] \overrightarrow{\mathbf{v}}^{l} u_{h}\right\|_{L^{2}\left(T_{i}^{h}\right)}\right) \\
\cdot\left\|\mathbf{A}_{h}\right\|_{L^{\infty}\left(T^{h}\right)}^{1 / 2}\left\|\nabla_{s_{h}}\left(\bar{R} \phi_{T_{i}^{h}}\right)\right\|_{L^{2}\left(T_{i}^{h}\right)} \\
\leq c\left(\left\|u-u_{h}^{l}\right\|_{H^{1}\left(T_{i}\right)}+\left\|\mathbf{C}_{h} \nabla_{s_{h}} u_{h}\right\|_{L^{2}\left(T_{i}^{h}\right)}+\left\|\sqrt{\mu_{h}}\left[\mathbf{I}-\mathbf{B}_{h}\right] \overrightarrow{\mathbf{v}}^{l} u_{h}\right\|_{L^{2}\left(T_{i}^{h}\right)}\right) \\
\cdot\left\|\mathbf{A}_{h}\right\|_{L^{\infty}\left(T_{i}^{h}\right)}^{1 / 2} h_{i}^{-1}\|\bar{R}\|_{L^{2}\left(T_{i}^{h}\right)}
\end{gathered}
$$

where the constant $c$ only depends on the shape regularity of $\mathcal{T}^{h}$.

Use Theorem 2.2 in [2] again, we then get

$$
\begin{aligned}
\|\bar{R}\|_{L^{2}\left(T_{i}^{h}\right)}^{2} & \leq c\left\|\bar{R} \sqrt{\phi_{T_{i}^{h}}}\right\|_{L^{2}\left(T_{i}^{h}\right)}^{2} \\
& \leq c\left(\int_{T_{i}^{h}} R \bar{R} \phi_{T_{i}^{h}} d s_{h}-\int_{T_{i}^{h}} \bar{R}(R-\bar{R}) \phi_{T_{i}^{h}} d s_{h}\right) \\
& \leq c\left(\int_{T_{i}^{h}} R \bar{R} \phi_{T_{i}^{h}} d s_{h}+\|R-\bar{R}\|_{L^{2}\left(T_{i}^{h}\right)}\left\|\bar{R} \phi_{T_{i}^{h}}\right\|_{L^{2}\left(T_{i}^{h}\right)}\right) \\
& \leq c\left(\int_{T_{i}^{h}} R \bar{R} \phi_{T_{i}^{h}} d s_{h}+\|R-\bar{R}\|_{L^{2}\left(T_{i}^{h}\right)}\|\bar{R}\|_{L^{2}\left(T_{i}^{h}\right)}\right) .
\end{aligned}
$$

Combination of (43) and (44) results in

$$
\begin{aligned}
\|\bar{R}\|_{L^{2}\left(T_{i}^{h}\right)}^{2} \leq c \| & \bar{R} \|_{L^{2}\left(T_{i}^{h}\right)}\left(\|R-\bar{R}\|_{L^{2}\left(T_{i}^{h}\right)}+h_{i}^{-1}\left\|\mathbf{A}_{h}\right\|_{L^{\infty}\left(T_{i}^{h}\right)}^{1 / 2}\left(\left\|u-u_{h}^{l}\right\|_{H^{1}\left(T_{i}\right)}\right.\right. \\
& \left.\left.+\left\|\mathbf{C}_{h} \nabla_{s_{h}} u_{h}\right\|_{L^{2}\left(T_{i}^{h}\right)}+\left\|\sqrt{\mu_{h}}\left(\mathbf{I}-\mathbf{B}_{h}\right) \overrightarrow{\mathbf{v}} u_{h}\right\|_{L^{2}\left(T_{i}^{h}\right)}\right)\right)
\end{aligned}
$$

Dividing (45) by $\|\bar{R}\|_{L^{2}\left(T_{i}^{h}\right)}$ and applying the triangle inequality, we immediately get

$$
\begin{aligned}
h_{i}\|R\|_{L^{2}\left(T_{i}^{h}\right)} \leq c( & h_{i}\|R-\bar{R}\|_{L^{2}\left(T_{i}^{h}\right)}+\left\|\mathbf{A}_{h}\right\|_{L^{\infty}\left(T_{i}^{h}\right)}^{1 / 2}\left(\left\|u-u_{h}^{l}\right\|_{H^{1}\left(T_{i}\right)}\right. \\
& \left.\left.+\left\|\mathbf{C}_{h} \nabla_{s_{h}} u_{h}\right\|_{L^{2}\left(T_{i}^{h}\right)}+\left\|\sqrt{\mu_{h}}\left[\mathbf{I}-\mathbf{B}_{h}\right] \overrightarrow{\mathbf{v}} u_{h}\right\|_{L^{2}\left(T_{i}^{h}\right)}\right)\right) .
\end{aligned}
$$

Next, we try to bound the edge residual $\|r\|_{L^{2}(e)}$ where $e$ is an edge shared by the triangle $T_{i}^{h}$ and one of its neighbors, says $T_{j}^{h}$. Let $K^{h}=\bar{T}_{i}^{h} \cup \bar{T}_{j}^{h}$, and correspondingly $K=\bar{T}_{i} \cup \bar{T}_{j}$, let $\phi_{e}$ be the edge bubble function [39] over $K^{h}$, i.e. $\left.\phi_{e}\right|_{\partial K^{h}}=0$ and $\phi_{e}=0$ outside $K^{h}$, and also let $\bar{r}$ be a piecewise linear approximation to $r$ (i.e., $\bar{r} \in \mathcal{U}$ ). Taking $\psi=\bar{r} \phi_{e}$ in (24), and applying the 
Poincare inequality and Theorem 2.4 in [2], we obtain

$$
\begin{aligned}
\left|\int_{e} r \bar{r} \phi_{e} d \gamma_{h}\right|=\mid & -\int_{K} a \nabla_{s}\left(u-u_{h}^{l}\right) \cdot \nabla_{s}\left(\bar{r}^{l} \phi_{e}^{l}\right) d s+\int_{K}\left(u-u_{h}^{l}\right) \overrightarrow{\mathbf{v}} \cdot \nabla_{s}\left(\bar{r}^{l} \phi_{e}^{l}\right) d s \\
& -\int_{K} b\left(u-u_{h}^{l}\right) \bar{r}^{l} \phi_{e}^{l} d s+\int_{K^{h}} R \bar{r} \phi_{e} d s_{h} \\
& -\int_{K} a\left(\mathbf{P}-\mathbf{A}_{h}^{l}\right) \nabla_{s} u_{h}^{l} \nabla_{s}\left(\bar{r}^{l} \phi_{e}^{l}\right) d s+\int_{K}\left(\mathbf{I}-\mathbf{B}_{h}^{l}\right)\left(\overrightarrow{\mathbf{v}} \nabla_{s}\left(\bar{r}^{l} \phi_{e}^{l}\right)\right) u_{h}^{l} d s \mid \\
\leq c & \left(\|R\|_{L^{2}\left(K^{h}\right)} h_{i}^{1 / 2}\|\bar{r}\|_{L^{2}(e)}+\left(\left\|u-u_{h}^{l}\right\|_{H^{1}(K)}+\left\|\mathbf{C}_{h} \nabla_{s_{h}} u_{h}\right\|_{L^{2}\left(K^{h}\right)}\right.\right. \\
& \left.\left.+\left\|\sqrt{\mu_{h}}\left(\mathbf{I}-\mathbf{B}_{h}\right) \overrightarrow{\mathbf{v}}^{l} u_{h}\right\|_{L^{2}\left(K^{h}\right)}\right)\left\|\mathbf{A}_{h}\right\|_{L^{\infty}\left(K^{h}\right)}^{1 / 2}\left\|\nabla_{s_{h}}\left(\bar{r} \phi_{e}\right)\right\|_{L^{2}\left(K^{h}\right)}\right)
\end{aligned}
$$

where the constant $c$ only depends on the shape regularity of the mesh. Again, using Theorem 2.4 in [2], we have

$$
\left\|\nabla_{s_{h}}\left(\bar{r} \phi_{e}\right)\right\|_{L^{2}\left(K^{h}\right)} \leq c h_{i}^{-1 / 2}\|\bar{r}\|_{L^{2}(e)}
$$

and

$$
\|\bar{r}\|_{L^{2}(e)}^{2} \leq c \int_{e} \bar{r}^{2} \phi_{e} d \gamma_{h} \leq c\left(\int_{e} r \bar{r} \phi_{e} d \gamma_{h}+\|r-\bar{r}\|_{L^{2}(e)} h_{i}^{1 / 2}\|\bar{r}\|_{L^{2}(e)}\right) .
$$

Combining (47)-(49), we then get

$$
\begin{aligned}
& h_{T_{i}^{h}}^{1 / 2}\|\bar{r}\|_{L^{2}(e)}^{2} \leq c\left(\| \mathbf { A } _ { h } \| _ { L ^ { \infty } ( K ^ { h } ) } ^ { 1 / 2 } \left(\left\|u-u_{h}^{l}\right\|_{H^{1}(K)}+\left\|\mathbf{C}_{h} \nabla_{s_{h}} u_{h}\right\|_{L^{2}\left(K^{h}\right)}\right.\right. \\
& \left.\left.\quad+\left\|\sqrt{\mu_{h}}\left(\mathbf{I}-\mathbf{B}_{h}\right) \overrightarrow{\mathbf{v}}^{l} u_{h}\right\|_{L^{2}\left(K^{h}\right)}\right)+h_{i}\|R\|_{L^{2}\left(K^{h}\right)}+h_{i}^{1 / 2}\|r-\bar{r}\|_{L^{2}(e)}\right)\|\bar{r}\|_{L^{2}(e)} .
\end{aligned}
$$

and consequently

$$
\begin{aligned}
& h_{T_{i}^{h}}^{1 / 2}\|r\|_{L^{2}(e)} \leq c\left(\| \mathbf { A } _ { h } \| _ { L ^ { \infty } ( K ^ { h } ) } ^ { 1 / 2 } \left(\left\|u-u_{h}^{l}\right\|_{H^{1}(K)}+\left\|\mathbf{C}_{h} \nabla_{s_{h}} u_{h}\right\|_{L^{2}\left(K^{h}\right)}\right.\right. \\
& \left.\left.\quad+\left\|\sqrt{\mu_{h}}\left(\mathbf{I}-\mathbf{B}_{h}\right) \overrightarrow{\mathbf{v}}^{l} u_{h}\right\|_{L^{2}\left(K^{h}\right)}\right)+h_{i}\|R\|_{L^{2}\left(K^{h}\right)}+h_{i}^{1 / 2}\|r-\bar{r}\|_{L^{2}(e)}\right) .
\end{aligned}
$$

Notice that $\mathbf{R}_{T_{i}^{h}, 2}$ and $\mathbf{R}_{T_{i}^{h}, 3}$ are higher-order terms compared with $\mathbf{R}_{T_{i}^{h}, 1}$ from the discussion in Remark 5, and we obtain the efficiency relation (42) directly from (46) and (51).

Remark 7 It is obvious that $\mathbf{C}_{h}$ and $\mathbf{I}-\mathbf{B}$ are of high order $\left(\geq h_{i}^{2}\right)$. We also can pick $\bar{R}$ and $\bar{r}$ properly such that the last two terms in (42) are again of high order $\left(\geq h_{i}^{1.5}\right)$, so that the dominant term in (42) is $\left\|u-u_{h}^{l}\right\|_{H^{1}\left(T_{i}\right)}$.

Remark 8 Actually, we would like to note that the same theoretical results can be obtained for closed surfaces $(\partial \mathbf{S}=\varnothing)$ with similar analysis as we did in the previous sections. 


\section{Numerical Experiments}

In this section, several numerical experiments are presented to verify the reliability and efficiency of the a posteriori estimator proposed in the previous section. All experiments are performed for the model equation (1) with a given exact solution $u(\mathbf{x})$. Boundary conditions are set correspondingly if $\partial \mathbf{S} \neq \varnothing$. In our experiments, the initial meshes are relatively uniform meshes; they are generated and optimized using the so-called constrained centroidal Voronoi Delaunay triangulation (CCVDT) algorithm [16] with a constant density function. The mesh refinement at each level is done by applying the marking strategy used in $[15,37]$ with the parameter $\theta=0.3(\theta$ is used to control the refinement process [21]). The refinement process stops after the number of nodes reaches 30,000 for each of the examples. We set $\beta_{1}^{*}=\beta_{2}^{*}=1$ in (39) for our numerical experiments. Recently, the study of robustness of a posteriori error estimates also attracted much attention, i.e., whether the constants in the a posteriori estimate are almost uniform for a class of similar problems. We will also numerically address this problem.

\subsection{Example 1: Half-sphere}

In this experiment, the surface $\mathbf{S}$ is taken to be the northern half of the unit sphere, i.e.,

$$
\mathbf{S}=\left\{\mathbf{x} \in \mathbb{R}^{3} \mid x_{1}^{2}+x_{2}^{2}+x_{3}^{2}=1, x_{3} \geq 0\right\},
$$

and its boundary is given by

$$
\partial \mathbf{S}=\left\{\left(x_{1}, x_{2}, 0\right) \mid x_{1}^{2}+x_{2}^{2}=0\right\} .
$$

The outer normal at $\mathbf{x} \in \mathbf{S}$ is simply $\left(x_{1}, x_{2}, x_{3}\right)$. We set $a(\mathbf{x})=1, \overrightarrow{\mathbf{v}}(\mathbf{x})=$ $(1,2,3), b(\mathbf{x})=1$, and the exact solution $u$ is chosen to be

$$
u(\mathbf{x})=\frac{1}{x_{1}^{2}+x_{2}^{2}+\left(1-x_{3}\right)^{2}+0.02}
$$

Clearly $u$ has a peak at $(0,0,1)$, the top of the half sphere. The first experiment takes all the coefficients to be constant. The initial mesh and the refined meshes at steps 12 and 16 are shown in Fig. 2. It can be easily seen that the meshes around the peak of the exact solution are well refined. In Fig. 3(left), we draw the $L^{2}$ and energy-norm errors of the approximate solution $u_{h}$ at all steps and also the a posteriori error estimate $\eta_{\mathcal{T}^{h}}$, along with some reference slopes. From Fig. 3 it can be concluded that the approximate solutions are of the second-order convergence when measured under $L^{2}$ norm and the first-order convergence under energy norm. It is also obvious that our error estimator has the same convergence rate as the energy-norm error, see Fig. 3(right) 

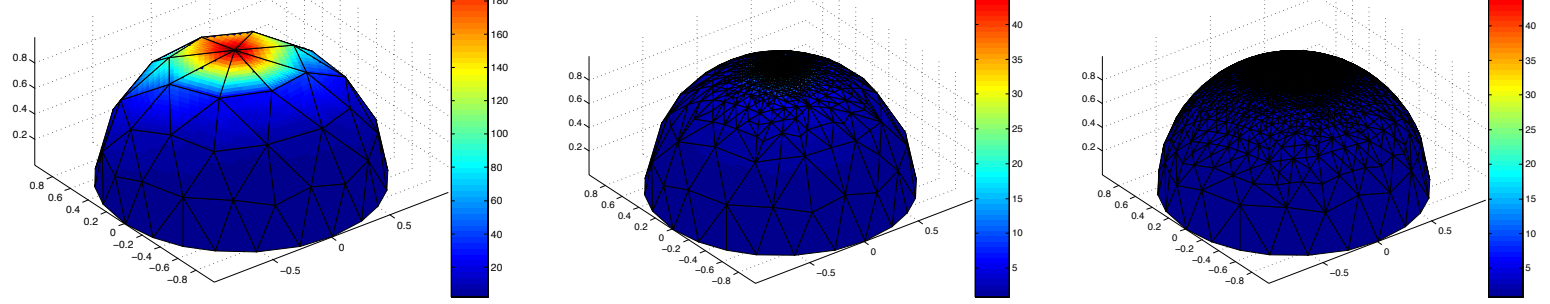

Fig. 2. (From left to right) The initial mesh with 64 nodes and adaptively refined meshes with 817 nodes (Level 12) and 4098 nodes (Level 16) for Example 1.
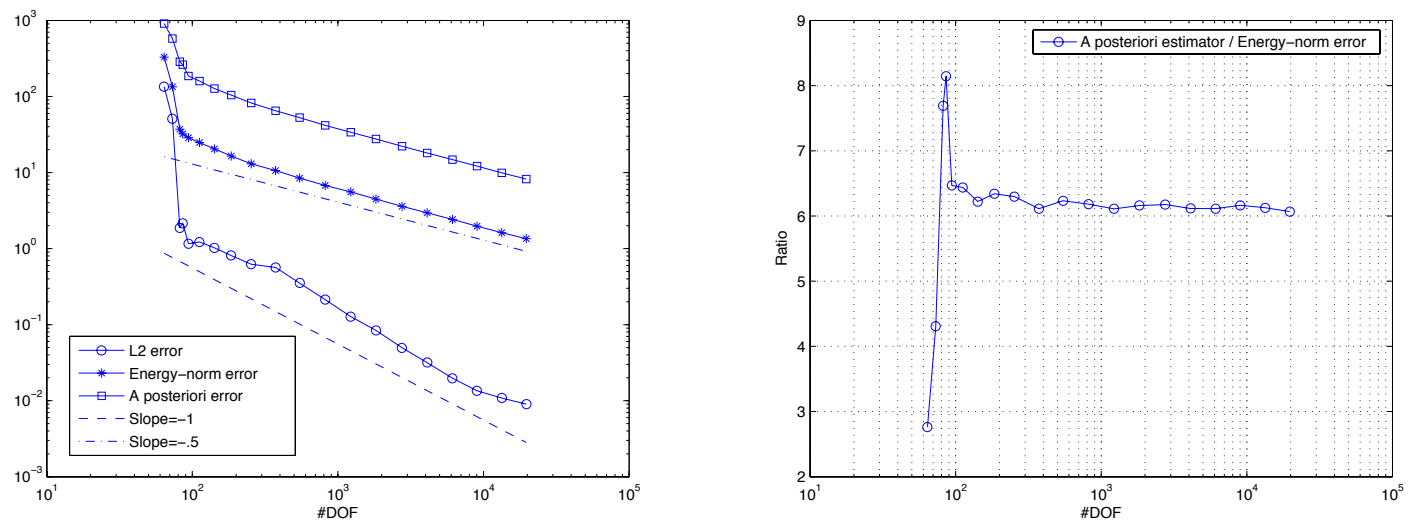

Fig. 3. Left: comparison of the $L^{2}$, Energy-norm errors and the a posteriori estimator $\eta_{\mathcal{T}^{h}}$ at all levels for Example 1; right: ratio between the error estimator and the exact energy-norm error for Example 1.

for information on the the ratio between the error estimator and the energynorm error at each step of refinements. From this figure, we can easily observe that the ratio quickly becomes stable after the oscillations at the first few refinements and converges to a constant around 6.1.

\subsection{Example 2: Cornered surface}

The surface $\mathbf{S}$ is now selected to be

$$
\mathbf{S}=\left\{\mathbf{x} \in \mathbb{R}^{3} \mid\left(x_{3}-x_{2}^{2}\right)^{2}+x_{1}^{2}+x_{2}^{2}=1, x_{3} \geq x_{2}^{2}, x_{1} \geq 0 \text { or } x_{2} \geq 0\right\}
$$

with the boundary

$$
\begin{aligned}
\partial \mathbf{S}= & \left\{\left(x_{1}, x_{2}, x_{2}^{2}+\sqrt{1-x_{1}^{2}+x_{2}^{2}}\right) \mid x_{1}^{2}+x_{2}^{2}=1, x_{1} \geq 0 \text { or } x_{2} \geq 0\right\} \\
& \cup\left\{\left(0, x_{2}, x_{2}^{2}+\sqrt{1+x_{2}^{2}}\right) \mid-1 \leq x_{2} \leq 0\right\} \cup\left\{\left(x_{1}, 0, \sqrt{1-x_{1}^{2}}\right) \mid-1 \leq x_{1} \leq 0\right\}
\end{aligned}
$$

Clearly, the boundary of $\mathbf{S}$ is not smooth at $(0,0,1)$. The outer normal at $\mathbf{x} \in \mathbf{S}$ is now given by $\overrightarrow{\mathbf{n}}(\mathbf{x})=\vec{t} /\|\vec{t}\|$ with $\vec{t}=\left(x_{1}, x_{2}\left(1-2\left(x_{3}-x_{2}^{2}\right)\right), x_{3}-x_{2}^{2}\right)$. 
We use variant coefficients $a(\mathbf{x})=2+x_{1} x_{2}, \overrightarrow{\mathbf{v}}(\mathbf{x})=\left(1+x_{1}, 2+x_{2}, 3+x_{3}\right)$ and $b(\mathbf{x})=1$. The exact solution $u$ is set again to be

$$
u(\mathbf{x})=\frac{1}{x_{1}^{2}+x_{2}^{2}+\left(1-x_{3}\right)^{2}+0.02} .
$$

Then the peak of error occurs at the corner $(0,0,1)$. In this experiment, we
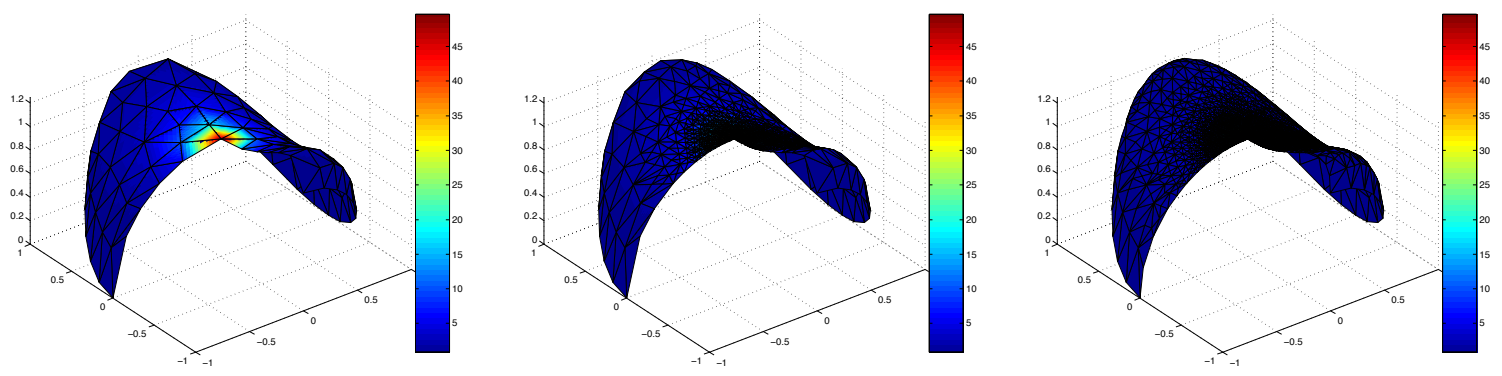

Fig. 4. (From left to right) The initial mesh with 108 nodes and adaptively refined meshes with 929 nodes (Level 11) and 3067 nodes (Level 14) for Example 2.
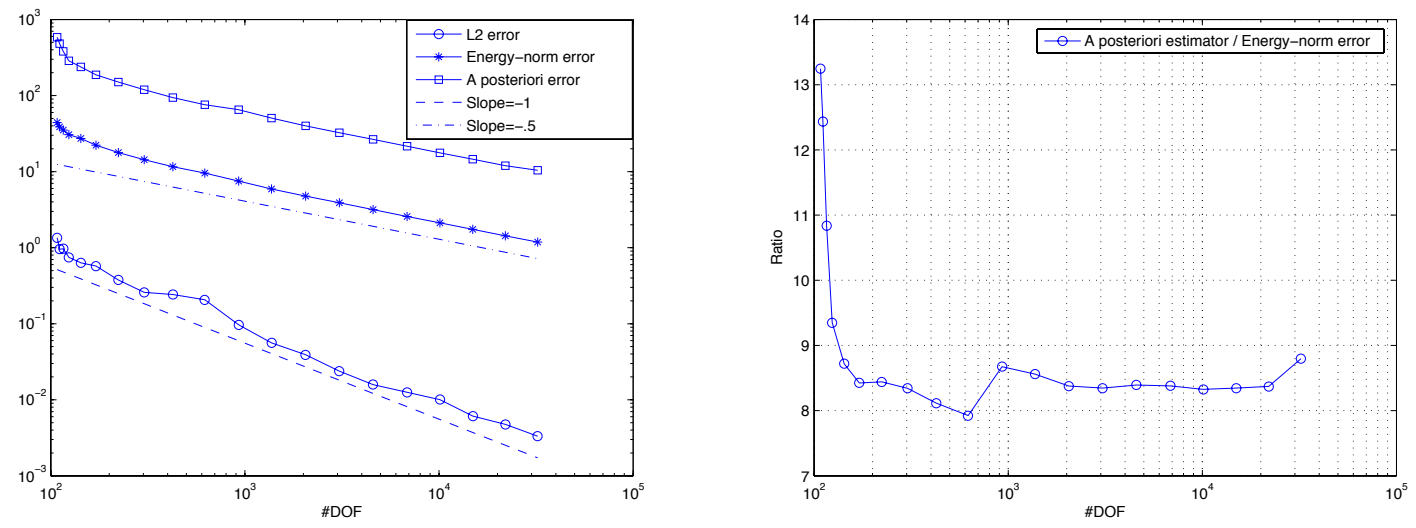

Fig. 5. Left: comparison of the $L^{2}$, Energy-norm errors and the a posteriori estimator $\eta_{\mathcal{T}^{h}}$ at all levels for Example 2; right: ratio between the error estimator and the exact energy-norm error for Example 2.

choose coefficients $a$ and $\overrightarrow{\mathbf{v}}$ to be dependent on $\mathbf{x}$, and the boundary is not globally smooth as that in experiment 1 (there are some corner points). We can see that the a posteriori estimate is still very effective, as shown in Fig. 4, where the meshes around the peak of exact solution are refined much more heavily, as expected. In Fig. 5(left), we see that the estimator $\eta_{\mathcal{T} h}$ has the same convergence rate as the energy-norm error, and the $L^{2}$ error maintains a second-order convergence. And as in experiment 1, the Fig. 5(right) gives us a steady almost-constant ratio between the error estimator and the energy-norm error, after oscillations of the first few steps of mesh refinement. Notice that the ratio is around 8.3 for this example which is quite close to that of Example 1 . 


\subsection{Example 3: Torus}

In the third experiment, the surface $\mathbf{S}$ is taken to be a torus such as

$$
\mathbf{S}=\left\{\mathbf{x} \in \mathbb{R}^{3} \mid\left(x_{1}-\frac{\left(r_{1}+r_{2}\right) x_{1}}{2 \rho}\right)^{2}+\left(x_{2}-\frac{\left(r_{1}+r_{2}\right) x_{2}}{2 \rho}\right)^{2}+x_{3}^{2}=\frac{\left(r_{2}-r_{1}\right)^{2}}{4}\right\}
$$

where $\rho=\sqrt{x_{1}^{2}+x_{2}^{2}}, r_{1}=0.5$, and $r_{2}=1$. Clearly, this surface has no boundary, and we will show that our estimator still works well.

The outer normal at $\mathbf{x} \in \mathbf{S}$ is given by $\overrightarrow{\mathbf{n}}(\mathbf{x})=\vec{t} /|\vec{t}|$ with

$$
\vec{t}=\left(\begin{array}{c}
\left(x_{1}-\tilde{x}_{1}\right)\left(1.0-\frac{r_{1}+r_{2}}{2 \rho}+\frac{\left(r_{1}+r_{2}\right) x_{1}^{2}}{2 \rho^{3}}\right)+\left(x_{2}-\tilde{x}_{2}\right)\left(\frac{\left(r_{1}+r_{2}\right) x_{2} x_{1}}{2 \rho^{3}}\right) \\
\left(x_{2}-\tilde{x}_{2}\right)\left(1.0-\frac{r_{1}+r_{2}}{2 \rho}+\frac{\left(r_{1}+r_{2}\right) x_{2}^{2}}{2 \rho^{3}}\right)+\left(x_{1}-\tilde{x}_{1}\right)\left(\frac{\left(r_{1}+r_{2}\right) x_{2} x_{1}}{2 \rho^{3}}\right)
\end{array}\right)
$$

where $\tilde{x}_{1}=\left(r_{1}+r_{2}\right) x_{1} / 2 \rho, \tilde{x}_{2}=\left(r_{1}+r_{2}\right) x_{2} / 2 \rho$. We let $a(\mathbf{x})=1+x_{1}^{2}$, $\overrightarrow{\mathbf{v}}=(0,0,0)$ and $b(\mathbf{x})=1+x_{1}^{2}+x_{2}^{2}+x_{3}^{2}$, and the exact solution $u$ is set to be

$$
u(\mathbf{x})=e^{\frac{1}{\left(x_{1}+1\right)^{2}+x_{2}^{2}+x_{3}^{2}+0.25}} .
$$

Obviously $u$ has a peak at $(-1,0,0)$. Fig. 6 shows that the meshes around

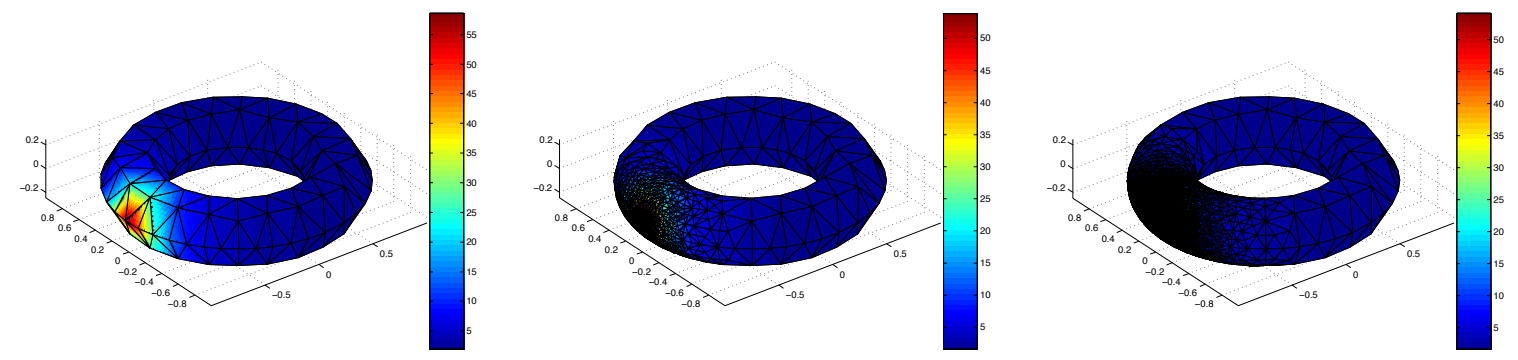

Fig. 6. (From left to right) The initial mesh with 146 nodes and adaptively refined meshes with 869 nodes (Level 9) and 4190 nodes (Level 13) for Example 3.

the peak are well-refined. Fig. 7 verifies the expected convergence rates of $L^{2}$, energy-norm errors and the a posteriori error estimate, as well as the almost-constant ratio between the error estimator $\eta_{\mathcal{T}^{h}}$ and the energy-norm error. More specifically, the ratio stays around 7.5 with small perturbations that is again quite close to that of Example 1 and Example 2. This numerical observation implies that the proposed a posteriori error estimate is also quite robust in applications. In this experiment, we in particular show that the proposed error estimator still behaves very well when the domain is a closed surface.

Remark 9 The robustness of the derived a posterior error estimator is numerically demonstrated through the above examples, while theoretical analysis 

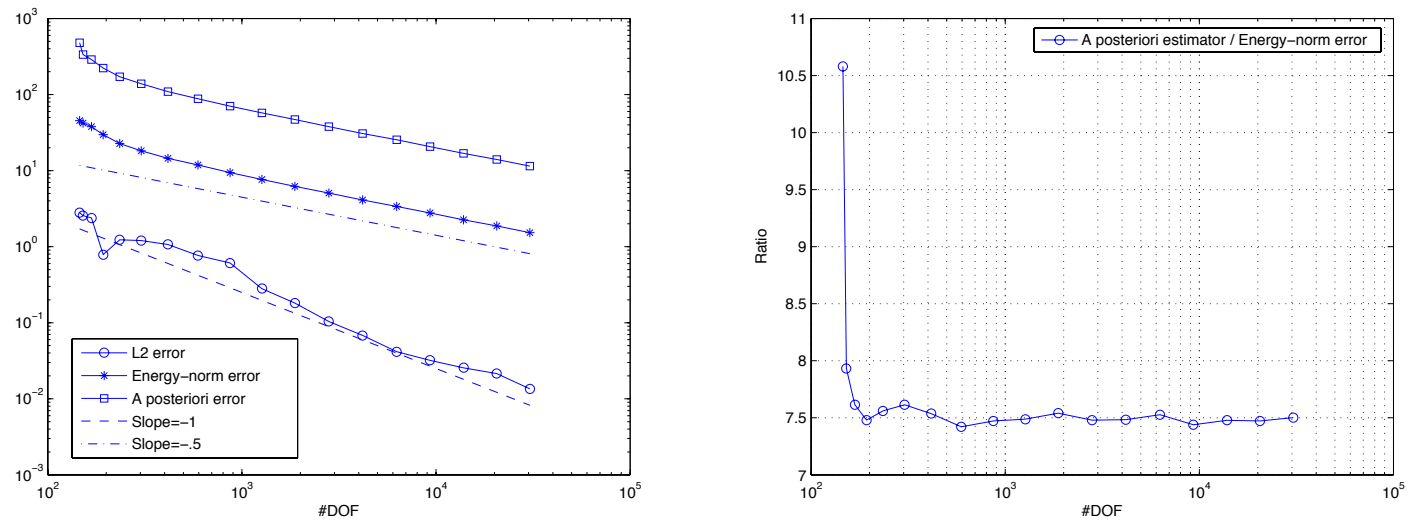

Fig. 7. Left: comparison of the $L^{2}$, Energy-norm errors and the a posteriori estimator $\eta_{\mathcal{T}^{h}}$ at all levels for Example 3; right: ratio between the error estimator and the exact energy-norm error for Example 3.

still remains open. Readers can refer to [36, 41] for more information on planar problems.

\section{Conclusions}

In this paper, we derive a residual-based explicit a posteriori error estimate for the finite volume discretization of elliptic partial differential equations defined on smooth surfaces in $\mathbb{R}^{3}$. We rigorously prove both the reliability and the efficiency of the proposed error estimator and verify the theoretical results through numerical examples. The numerical results also clearly demonstrate the robustness of the proposed error estimator. We note that the central discretization scheme will not be appropriate when the equation (1) becomes convection-dominated (i.e., $|a| /|\overrightarrow{\mathbf{v}}|<<1$ ). Under that case an up-wind approximation of the convection term is needed to avoid non-physical oscillations and similar analysis is still under our study. The on-going and future works also includes studying a posteriori error estimators on finite volume approximation for higher-order and time-dependent problems defined on surfaces.

\section{References}

[1] M. Afif, A. Bergam, Z. Mghazli and R. Verfürth, A posteriori estimators for the finite volume discretization of an elliptic problem, Numer. Algorithm, 34, pp. 127-136, 2003.

[2] M. Ainsworth and J. Oden, A Posteriori Error Estimation in Finite Element Analysis, Wiley, New York, 2002. 
[3] T. Apel And C. Pester, Clement-type interpolation on spherical domains-interpolation error estimates and application to a posteriori error estimation, IMA J. Numer. Anal., 25, pp. 310-336, 2005.

[4] I. Babuska And A. Miller, A feedback finite element method with a posteriori error estimates, Comput. Methods Appl. Mech. Engrg., 61, pp. 1-40, 1987.

[5] I. Babuska And W. C. Rheinboldt, Error estimates for adaptive finite element computations, SIAM J. Numer. Anal., 15, pp. 736-754, 1987.

[6] I. Babuska and W. C. Rheinboldt, A posteriori error estimates for the finite element method, Internat. J. Numer. Methods Engrg., 12, pp. 1597-1615, 1978.

[7] I. Babuska and W. C. Rheinboldt, A posteriori error analysis of finite element solutions for one-dimensional problems, SIAM J. Numer. Anal., 18, pp. 565-589, 1981.

[8] E. Bänsch, P. Morin And R. H. Nochetto (2005) A finite element method for surface diffusion: the parametric case, J. Comput. Phys., 203, pp. 321-343.

[9] S. Bartels, C. Carstensen and G. Dolzmann, Inhomogeneous Dirichlet conditions in a priori and a posteriori finite element error analysis, Numer. Math., 99, pp. 1-24, 2004.

[10] M. Bertalmio, L.-T. Cheng, S. Osher and G. Sapiro, Variational methods and partial differential equations on implicit surfaces, J. Comput. Phys., 174, pp. 759-780, 2001.

[11] C. Carstensen, All first-order averaging techniques for a posteriori finite element error control on unstructured grids are efficient and reliable, Math. Comp., 73, pp. 1153-1165, 2003.

[12] C. Carstensen, R. Lazarov And S. Tomov, Explicit and averaging a posteriori error estimates for adaptive finite volume methods, SIAM J. Numer. Anal., 42, pp. 2496-2521, 2005.

[13] S. Chou And Q. Li, Error estimates in $L^{2}, H^{1}$ and $L^{\infty}$ in covolume methods for elliptic and parabolic problems: a unified approach, Math. Comp., 69, pp. 103-120, 2000.

[14] A. Demlow, Local a posteriori estimates for pointwise gradient errors in finite element methods for elliptic problems, Math. Comp., 76, pp. 19-42, 2007.

[15] A. Demlow and G. Dziuk, An adaptive finite element method for the Laplace-Beltrami operator on implicitly defined surfaces, SIAM J. Numer. Anal., 45, pp. 421-442, 2007.

[16] Q. Du, M. Gunzburger and L. Ju, Constrained centroidal Voronoi tessellations on general surfaces, SIAM J. Sci. Comput., 24, pp. 1488-1506, 2003.

[17] Q. Du, M. Gunzburger, And L. Ju, Voronoi-based finite volume methods, optimal Voronoi meshes and PDEs on the sphere, Comp. Meth. Appl. Mech. Engrg., 192, pp. 3933-3957, 2003.

[18] Q. DU AND L. JU, Finite volume methods on spheres and spherical 
centroidal Voronoi meshes, SIAM J. Numer. Anal., 43, pp. 1673-1692, 2005.

[19] Q. DU AND L. JU A finite volume method on general surfaces and its error estimates, submitted.

[20] Q. Du, L. Ju And L. Tian, Analysis of a mixed finite volume discretization for fourth-order equations on general surfaces, IMA J. Numer. Anal., to appear.

[21] W. Dörfler, A convergent adpative algorithm for Poisson's equation, SIAM J. Numer. Anal., 33, pp. 1106-1124, 1996.

[22] G. DzIUK, Finite elements for the Beltrami operator on arbitrary surfaces, Partial Differential Equations and Calculus of Variations, ed. by S. Hildebrandt and R. Leis, Lecture Notes in Mathematics, 1357, Springer, Berlin, pp. 142-155, 1988.

[23] G. Dziuk and C. M. Elliot, Finite elements on evolving surfaces, IMA J. Numer. Anal., 27, 262-292, 2007.

[24] G. Dziuk and C. M. Elliot, Surface finite elements for parabolic equations, J. Comput. Math., 25, pp. 385-407, 2007.

[25] P. Evans, L. Schwartz And R. Roy, Steady and unsteady solutions for coating flow on a rotating horizontal cylinder: Two-dimensional theoretical and numerical modeling, Phys. of Fliuds, 16, pp. 2742-2756., 2004

[26] E. Hebey, Sobolev Spaces on Riemannian Manifolds, Springer, Berlin, 1991.

[27] R. Heikes AND D. Randall, Numerical integration of the shallowwater equations on a twisted icosahedral grid, Monthly Weather Review, 123, pp. 1862-1887, 1995.

[28] M. Holst, Adaptive numerical treatment of elliptic system on manifold, Adv. Comput. Math., 15, pp. 139-191, 2001.

[29] L. Ju, M. Gunzburger And W. Zhao, Adaptive finite element methods for elliptic PDEs based on conforming centroidal voronoi-delaunay triangulations, SIAM J. Sci. Comput., 28, pp. 2023-2053, 2003.

[30] G. Kanschat, Dominik Schötzau, Energy norm a posteriori error estimation for divergence-free discontinuous Galerkin approximations of the Navier-Stokes equations, International Journal for Numerical Methods in Fluids, 57, pp. 1093 - 1113, 2008.

[31] R. Lazarov and S. Tomov, A posteriori error estimates for finite volume element approximations of convection-diffusion-reaction equations, Comput. Geosciences, 6, pp. 483-503, 2002.

[32] P. Morin, R. H. Nochetto, and K. G. Siebert, Data oscillation and convergence of adaptive FEM, SIAM J. Numer. Anal., 38, pp. 466-488, 2000.

[33] F. Memoli, G. Sapiro, And S. Osher, Solving variational problems and partial differential equations mapping into general target manifolds, J. Comput. Phys., 195, pp. 263-292, 2004.

[34] F. Memoli, G. Sapiro, and P. Thompson, Implicit brain imaging, Human Brain Mapping, 23, pp. 179-188, 2004.

[35] T. Myers and J. Charpin, A mathematical model for atmospheric ice 
accretion and water flow on a cold surface, International Journal of Heat and Mass Transfer, 47, pp. 5483-5500, 2004.

[36] G. Sangalli, Robust a-posteriori estimator for advection-diffusionreaction problems, Math. Comp., 77, pp. 41-70, 2008.

[37] A. Schmidt A And K. Siebert, Design of Adaptive Finite Element Software: The Finite Element Toolbox ALBERTA, Lect. Notes Comp. Sci. Eng., 42, Springer, New York, 2005.

[38] H. Si AND K. GaerTner, Meshing piecewise linear complexes by constrained Delaunay tetrahedralizations, Proceedings of the 14th International Meshing Roundtable, pp. 147-163, 2005.

[39] R. Verfürth, A posteriori error estimators for the Stokes equations, Numer. Math., 55, pp. 309-325, 2005.

[40] R. VeRFüRTH, A review of a posteriori error estimation and adaptive mesh-refinement techniques, Wiley-Teubner, Chichester, UK, 1996.

[41] R. VERFÜRTH, Robust a posteriori error estimators for the singularly perturbed reaction-diffusion equation, Numerische Mathematik, 78, pp. 479493, 1998. 